\title{
Arreglo de fracturas geológicas en rocas miocénicas de la cuenca de México
}

\section{Alberto Vásquez-Serrano ${ }^{1, *}$, José Luis Arce-Saldaña ${ }^{1}$, Elizabeth Rangel-Granados ${ }^{1}$, Eric Morales-Casique ${ }^{2}$ y Sarah María Arroyo López ${ }^{1}$}

\author{
${ }^{1}$ Departamento de Procesos Litosféricos, Instituto de Geología, Universidad Nacional Autónoma de México, \\ Circuito de la Investigación Científica s/n, Ciudad Universitaria, Coyoacán, Ciudad de México, C.P. 04510, México. \\ ${ }^{2}$ Departamento de Dinámica Superficial, Instituto de Geología, Universidad Nacional Autónoma de México, \\ Circuito de la Investigación Científica s/n, Ciudad Universitaria, Coyoacán, Ciudad de México, C.P. 04510, México. \\ *alberto-vasquez@ciencias.unam.mx
}

\section{RESUMEN}

El estudio del arreglo de las fracturas es importante para el conocimiento del flujo de fluidos y tiene una amplia aplicación en áreas como: estabilidad de taludes, campos geotérmicos, acuíferos profundos y exploración de hidrocarburos. El arreglo de las fracturas es una característica fundamental de la distribución espacial de las fracturas, aunque su relación con el tipo de roca, fallas kilométricas y herencia estructural es poco conocida. La influencia de estas variables en el arreglo de las fracturas es explorada en este trabajo mediante un estudio detallado de fracturas en rocas miocénicas de la cuenca de México. Las rocas miocénicas en la cuenca de México están afectadas por tres sistemas de fracturas que tienen una orientación NE-SW, NNW-SSE y $\sim$ E-W. Las tres familias de fracturas se presentan con un arreglo en forma de grupos de fracturas, donde el grado de agrupamiento está relacionado con el tipo de roca que constituye la sucesión volcánica del Mioceno (lavas y depósitos volcaniclásticos). Adicionalmente, la intensidad de fracturas es mayor en las lavas y menor en los depósitos volcaniclásticos, y tiene una relación inversa con el grado de agrupamiento. La familia F1 (NE-SW) tiene un arreglo de fracturas que se pueden caracterizar con una distribución fractal a escala de metros. Mientras que las fracturas F2 (NNW-SSE) presentan un arreglo que se asocian con anisotropías mecánicas en rocas pre-miocénicas. Este fenómeno probablemente está relacionado con una herencia estructural y/o reactivación de estructuras. Finalmente, las fracturas F3 ( E-W) forman un arreglo que se puede asociar con una distribución espacial aleatoria.

Palabras clave: arreglo de fracturas; fractura geológica; análisis fractal; intensidad de fracturas; rocas del Mioceno.

\begin{abstract}
The study of fracture arrangement is important for the understanding of fluid flow and has a wide application in several areas as slope stability analysis, geothermal fields, deep aquifers and oil exploration. The facture arrangement is an essential characteristic of the spatial distribution of the fractures, although the relation of the spatial arrangement of fractures
\end{abstract}

with variables such as the rock type, the kilometric faults, and structural inheritance is unknowns. We explore de influence of these variables in the facture arrangement through of detail study of Miocene rocks in the Mexico basin. The Miocene rocks in the Mexico Basin are affected by three fracture systems oriented NE-SW, NNW-SSE and $\sim E-W$. All fracture families present a cluster array, where the degree of clustering is related to the rock type that constitute the Miocene volcanic succession (lava flows and volcaniclastic deposits). In addition, the intensity of fracturing is stronger in lava flows than the volcaniclastic deposits, with an inverse relationship to the degree of clustering (Lyapunov Exponent). The F1 family (NE-SW) has groups of fractures characterized by a fractal distribution in mesoscopic scale. Meanwhile, the fractures F2 (NNWSSE) present groups that are associated with mechanical anisotropy in pre-Miocene rocks. This phenomenon is related to structural inheritance and/or reactivation. Finally, the fractures $F 3(\sim E-W)$ form groups with a random distribution.

Key words: fracture arrangement; geologic fracture; fractal análisis; fracture intensity; Miocene rocks.

\section{INTRODUCCIÓN}

Las fracturas geológicas son estructuras ligeramente planas y relativamente continuas que pueden afectar a las rocas en diversas escalas, desde $\mathrm{cm}$ hasta cientos de $\mathrm{km}$ (Fossen, 2010). Estas estructuras pueden tener cierto desplazamiento perpendicular o paralelo a sus paredes, dependiendo si son extensionales (Modo I) o de cizalla (Modo II y III) respectivamente (Fossen, 2010). En algunos casos, existen fracturas que combinan extensión y cizalla llamadas híbridas o mixtas (Ramsey y Chester, 2004). Estos modos de fractura pueden darse bajo una mecánica de fractura lineal (frágil) o no lineal (dúctil) dependiendo de las condiciones de presión y temperatura (Fossen, 2010). Las fracturas que se forman en la corteza comúnmente suelen ser rellenas de material formando vetas, lo cual pone en evidencia el paso de fluidos a través de ellas (Davis y Reynolds, 1996). Un aspecto importante en el análisis de fracturas es el arreglo que presentan. El arreglo describe la posición en el espacio de las fracturas incluyendo 
su grado de agrupamiento (Laubach et al., 2018; Wang et al., 2019). El arreglo de las fracturas está íntimamente relacionado con la heterogeneidad estructural y la anisotropía de la corteza superior terrestre (Olson y Pollard, 1989; Bear et al., 2012; Laubach et al., 2018; Samsu et al., 2020). El conocimiento sobre el arreglo de las fracturas geológicas es fundamental en su aplicación para áreas como: sismología, inyección y extracción de fluidos en pozos profundos en la industria petrolera, sistemas geotérmicos y acuíferos profundos. A pesar de la importancia del arreglo de las fracturas, aún no se establece de manera clara su relación con el tipo de roca, la herencia estructural y la influencia de estructuras mayores (pliegues y fallas). En este trabajo se hace un análisis detallado de las fracturas con el propósito de conocer qué relación tiene el tipo de roca, la herencia estructural y las estructuras mayores en el arreglo de las fracturas. Nuestro estudio se realiza en rocas del Mioceno que afloran en tres sitios estratégicos dentro y en los límites de la cuenca de México: Sierra de Guadalupe, Tepoztlán y Malinalco (Figura 1). El conocimiento de estas rocas es fundamental para entender la contribución que tienen las fracturas en la generación de permeabilidad secundaria en acuíferos profundos de la Ciudad de México (Pérez-Cruz, 1988; Vásquez-Serrano et al., 2019; Arce et al., 2019). Nuestro análisis hace uso de herramientas fractales para conocer el arreglo de las fracturas a través de parámetros como la dimensión fractal de caja, dimensión de correlación y exponente de Lyapunov (Vásquez-Serrano et al., 2019). Así mismo, se introduce la aplicación del método de suma de correlación normalizada (SCN) expuesto en Marrett et al. (2018), el cual ha demostrado su eficiencia en el análisis del arreglo de fracturas. Los resultados de este trabajo podrán ser de gran interés en futuros estudios de permeabilidad y flujo subterráneo por debajo del subsuelo de la Cuenca de México.

\section{MARCO GEOLÓGICO}

La cuenca de México está localizada dentro del sector oriental de la Faja Volcánica Transmexicana (FVTM), un arco volcánico que tiene una longitud de $\sim 1000 \mathrm{~km}$ con un rumbo E-W. Dicho arco va desde las costas de Nayarit en el océano Pacífico hasta las costas de Veracruz en el Golfo de México (Figura 1a) (Ferrari et al., 2011). La FVTM es el resultado de la subducción de las placas oceánicas de Cocos y Rivera por debajo de la placa Norteamericana (Pardo y Suárez, 1995; Manea et al., 2013).

Muchos estudios geológicos y geofísicos han sido realizados dentro de la cuenca de México con el objetivo de conocer su estratigrafía, geología estructural y tectónica (Fries, 1956, 1960; Mooser, 1963, 1975; de Cserna et al., 1988; García-Palomo et al., 2008; Arce et al., 2019). Así mismo, se han hecho diversos estudios geológicos de detalle en las principales sierras que rodean a la cuenca de México (Osete et al., 2000; Arce et al., 2008; García-Palomo et al., 2008; Cadoux et al., 2011; Macias et al., 2012) y en el paquete fluvio-volcano-lacustre del Pleistoceno tardío-Holoceno (Avendaño-Villeda et al., 2018 Martínez-Abarca et al., 2019). Los datos obtenidos del paquete fluvio-volcano-lacustre han aportado datos valiosos de la estratigrafía más superficial y de las condiciones paleoambiantales de la cuenca de México (LozanoGarcía et al., 1993; Lozano-García y Ortega-Guerrero, 1998; Brown et al., 2012; Avendaño-Villeda et al., 2018 Martínez-Abarca et al., 2019). Adicionalmente, se han hecho estudios geocronológicos detallados en las rocas cortadas por los pozos profundos perforados en la Ciudad de México. Estos han ayudado a refinar la estratigrafía del subsuelo de la cuenca de México, con lo cual se han definido de mejor manera las relaciones estratigráficas con las rocas expuestas en superficie (Arce et al., 2013; 2015; 2019). Los pozos profundos perforados por Petróleos Mexicanos (PEMEX) en el siglo pasado y los recientemente perforados (pozo San Lorenzo Tezonco en 2012, pozos Agrícola Oriental 2B y 2C en 2016 y pozo Santa Catarina 3 A en 2017) por el Sistema de Aguas de la Ciudad de México, han sido de gran ayuda para conocer no sólo el tipo de roca que existe a profundidad, sino también la geometría del basamento Mesozoico, la definición de fallas cenozoicas kilométricas, y las condiciones hidrogeológicas bajo la Ciudad de México (Pérez-Cruz, 1988; Arce et al., 2015; Morales-Casique et al. 2014; Lezama-Campos et al., 2016; Morales- Casique et al. 2016; Morales-Casique et al. 2017; Vásquez-Serrano et al., 2019).

\section{Estratigrafía en el centro de la cuenca de México}

Las rocas más antiguas en la cuenca de México corresponden a rocas calcáreas que se correlacionan con las formaciones geológicas del Cretácico que afloran al sur de la cuenca, particularmente en el área de Cuernavaca-Tepoztlán (Figura 1b) (Fries, 1960; Pérez-Cruz, 1988; Arce et al., 2013, 2015). Cubriendo discordantemente a este basamento calcáreo se encuentran conglomerados calcáreos del Eoceno pertenecientes al Grupo Balsas (González-Torres et al., 2015) y rocas volcánicas del Eoceno-Oligoceno (Pérez-Cruz, 1988). Sobreyaciendo a estas rocas hay depósitos volcánicos del Mioceno (asociados con la actividad de la FVTM) expuestos en la Sierra de Guadalupe (García-Palomo et al., 2006), en los alrededores de Tepoztlán, Morelos (Lenhardt et al., 2010) y en el área de Malinalco, Estado de México (Figura 1b) (García-Palomo et al., 2000). Asimismo, rocas de esta misma edad han sido cortadas entre 1 y $2 \mathrm{~km}$ de profundidad por los pozos profundos Mixhuca, Tulyehualco, San Lorenzo Tezonco y Roma (Pérez-Cruz, 1988; Arce et al., 2013, 2015, 2019). Cubriendo a estas rocas existen depósitos volcánicos del Plioceno al Holoceno que afloran en la Sierra de las Cruces (Arce et al., 2008), Sierra Nevada (Macías et al., 2012) y Sierra Chichinautzin (Bloomfield, 1975; Wallace y Carmichael 1999; Márquez et al., 1999; Siebe, 2000; Siebe et al., 2005; Guilbaud et al., 2009, Agustín-Flores et al., 2011; Arce et al., 2015). Finalmente, en la cima de la columna existen sedimentos lacustres del Pleistoceno al Holoceno (Lozano-García et al., 1993; Lozano-García y Ortega-Guerrero, 1998; Brown et al., 2012; Lozano-García et al., 2017) intercalados con tefras de diferentes fuentes (Sierra de Santa Catarina, Sierra Nevada y Sierra Chichinautzin) (Figura 1).

\section{Fallas geológicas en la cuenca de México}

En la cuenca de México se han documentado por lo menos tres sistemas de fallas con cinemática normal: NNW-SSE, E-W y NE-SW (Figura 1b) (Alaniz-Álvarez et al., 1998; García-Palomo et al., 2008; Arce et al., 2019). Algunos de estos sistemas han sido reactivados durante la extensión cortical N-S activa en la FVTM (Alaniz-Álvarez et al., 2001, 2002). Estas familias de fallas están presentes en la mayor parte del centro de México dentro de la FVTM, siendo el sistema NNW-SSE el que fuera activo en el Oligoceno- Mioceno, y el sistema $\sim$ E-W el actualmente activo (Alaniz-Álvarez et al., 1998).

En la zona occidental y noroccidental de la cuenca de México, dentro de la Sierra de las Cruces y la parte occidental de la Sierra de Guadalupe (Figura 1c) se han documentado fallas importantes como: falla Otomí (E-W), fallas Río Hondo (ENE-WSW), fosa de Barrientos (NE-SW) y fallas Catedral (N-S). Así mismo, al norte y al sur de la Sierra Chichinautzin se han descrito fallas con orientación E-W, como falla Xochimilco y falla La Pera (Figura 1c); éstas se presentan como lineamientos observables en imágenes satelitales (Delgado-Granados et al., 1995; Alaniz-Álvarez y Nieto-Samaniego, 2005; Norini et al., 2006; García-Palomo et al., 2008; Arce et al., 2013; Lermo et al., 2016; Arce et al., 2019).

En el centro de la cuenca de México se han definido dos sistemas de fallas principales: uno, ENE-WSW, representado por lineamientos paralelos a la Sierra de Santa Catarina. A este sistema pertenece la 


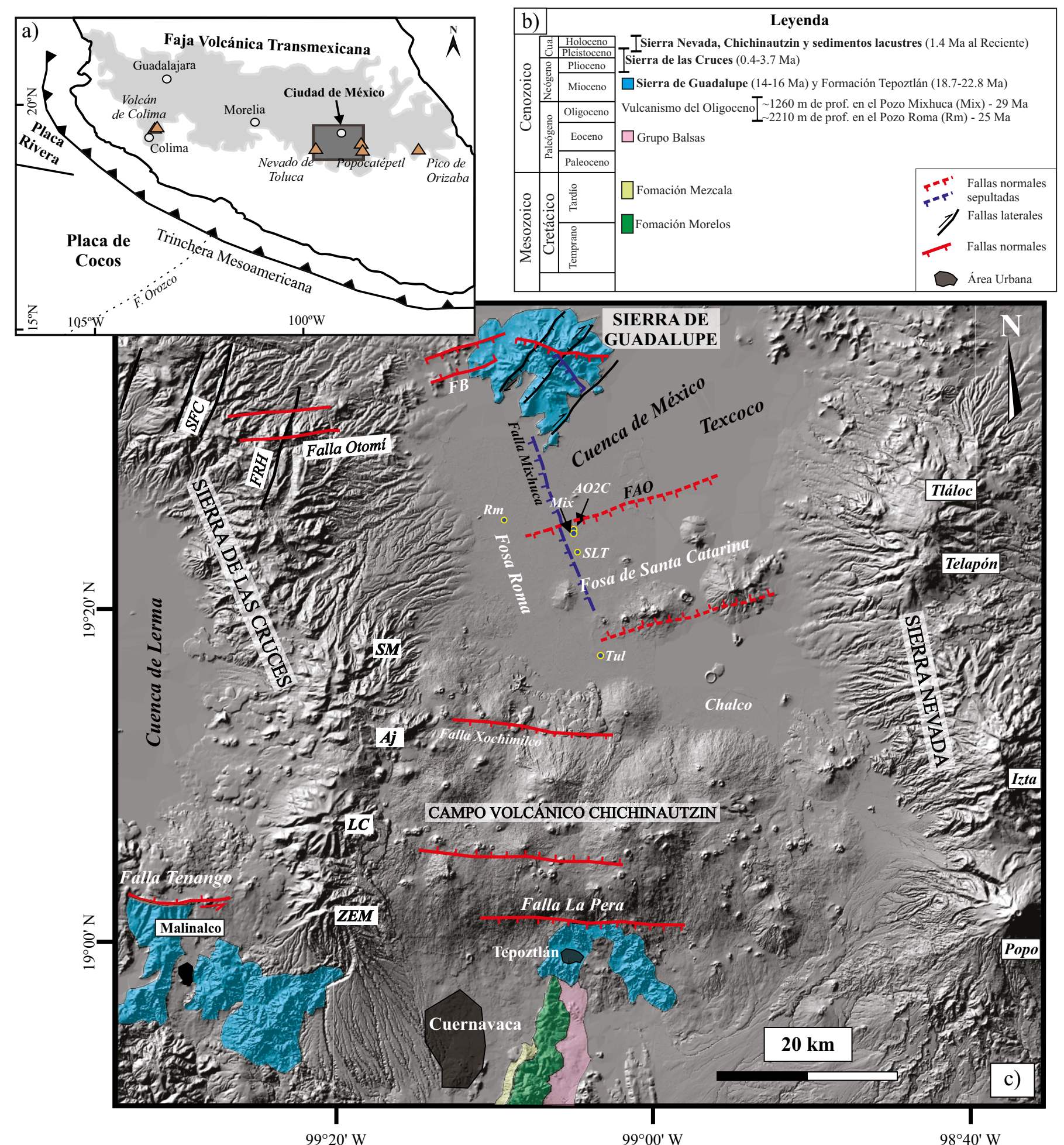

Figura1. a) Mapa tectónico del centro-sur de México donde se muestra a la Faja Volcánica Transmexicana y la ubicación de la cuenca de México en el cuadro gris (Modificado de Arce et al., 2013). b) Columna estratigráfica representativa de la cuenca de México. c) Modelo digital de elevación que representa el cuadro gris de inciso a, donde se aprecian las sierras volcánicas que rodean a la cuenca de México, las principales fallas geológicas reportadas en trabajos previos (Alaniz-Álvarez et al., 1998; García-Palomo et al., 2008; Arce et al., 2013; Lermo et al., 2016) y los afloramientos de las rocas del Mioceno estudiados; así mismo, se incluye la ubicación de los pozos: AO2C-Agrícola Oriental: 19²3'43.25"N, 99 5'11.01"W, Mix-Mixhuca: 19²3’19”N, 9905’17”W; SLT- San Lorenzo Tezonco: $19^{\circ} 22^{\prime} 44.18^{\circ}$ " N;

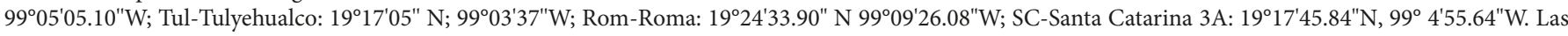
estructuras volcánicas son: SM-San Miguel, AJ-Ajusco, LC-La Corona, ZEM-Zempoala, Izta-Iztaccíhuatl, Popo-Popocatépetl (Arce et al., 2019; Macías et al., 2012) y estructuras tectónicas: FB-Fosa Barrientos, SFC-Sistema de Fallas Catedral, FRH-Falla Río Hondo (García-Palomo et al., 2008), FAO-Falla Agrícola Oriental (en este trabajo). Se indican las fuentes de las edades de las siguientes formaciones o rasgos topográficos: Lenhardt et al. (2010) para la Fm. Tepoztlán; Lozano-Barraza (1968) para la Sierra de Guadalupe y la compilación hecha por Arce et al. $(2008 ; 2019)$ para la Sierra de las Cruces y Cuenca de México. 
fosa de Santa Catarina, la cual tiene una orientación ENE-WSW y fue definida gracias a las columnas litológicas descritas en los pozos Mixhuca, San Lorenzo Tezonco y Tulyehualco (Arce et al. 2013; Vásquez-Serrano et al., 2019). El otro sistema de fallas que se observa dentro de la cuenca, tiene una orientación NNW-SSE y fue definido con base en los estudios realizados por Pérez-Cruz (1988), donde se determinó la variación de la cima del basamento Mesozoico. Gracias a dicha variación se pudo definir a la falla Mixhuca, la cual es de tipo normal y parece acomodar un desplazamiento importante de más de $3 \mathrm{~km}$ en la fosa tectónica Roma (Pérez-Cruz, 1988).

La actividad reciente del sistema de fallas $\sim \mathrm{E}-\mathrm{W}$ es confirmada por la sismicidad asociada con fallas normales que tienen una orientación ENE-WSW (Lermo et al., 2016). Estos eventos indican que este sistema es activo, congruente con lo observado en otras partes de la FVTM (Suter et al., 2001; Sunye-Puchol et al., 2015) y con la deformación actual del arco volcánico (Alaniz-Álvarez et al., 1998; Ferrari et al., 2011; Manea et al., 2013).

\section{METODOLOGÍA}

El análisis del arreglo de las fracturas realizado en este trabajo, contempla una medición sistemática de las fracturas en tres sitios estratégicos (Figura 1c): Sierra de Guadalupe (al norte de la Ciudad de México), Tepoztlán (al sur de la cuenca de México) y Malinalco (al surponiente de la cuenca de México), donde afloran rocas volcánicas y volcaniclásticas del Mioceno (Lenhardt et al., 2010; Arce et al., 2019). Dichos sitios son relevantes debido a que son un análogo expuesto de rocas volcánicas fracturadas ubicadas en el subsuelo de la Ciudad de México, las cuales seguramente tienen un rol importante en la generación de acuíferos profundos (Morales-Casique et al. 2014; Lezama-Campos et al., 2016; Morales-Casique et al. 2016; MoralesCasique et al. 2017). En cada uno de los sitios estratégicos se buscaron afloramientos de rocas fracturadas que permitieran medir de manera extensa el espaciamiento entre las fracturas (distancia entre dos fracturas) a través de líneas de muestreo perpendiculares al rumbo de las estructuras (Figura 2. La Pointe y Hudson, 1985). La selección de los afloramientos se realizó siguiendo las recomendaciones propuestas por Vásquez-Serrano (2013), siendo la más importante aquella relacionada con el reflejo del grado de agrupamiento de las fracturas. Así mismo, se tomaron de manera independiente datos de apertura de las fracturas en cada afloramiento, tratando de tomar la máxima apertura. Esta variable se define como la distancia entre las paredes de

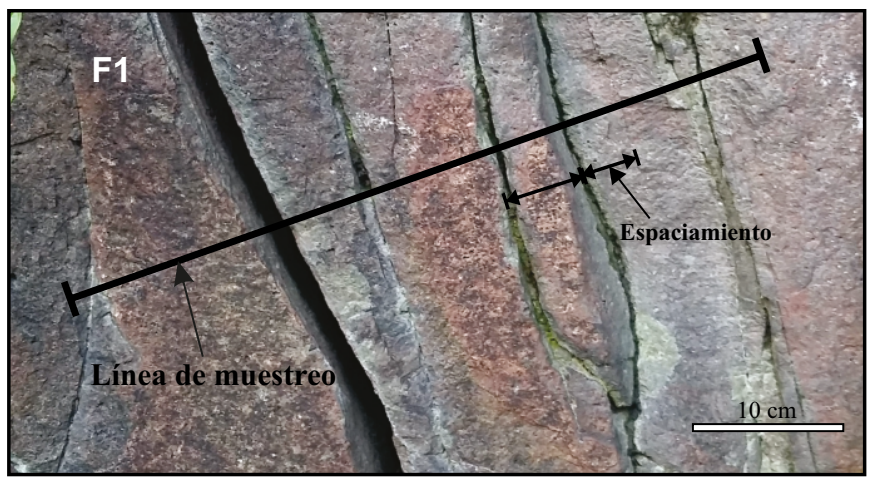

Figura 2. Método de obtención de los datos de espaciamiento (distancia entre 2 fracturas) en una familia de fracturas (grupo de fracturas paralelas) a través de una línea de muestreo. Afloramiento de fracturas NE-SW en la Sierra de Guadalupe en el centro de la cuenca de México. una fractura. La apertura fue medida en fracturas hibridas paralelas al rumbo preferencial de los sistemas principales de fallas. La cantidad de extensión y cizalla de las fracturas híbridas no es cuantificada en nuestro trabajo. Dentro de los datos de apertura se incluyeron también fracturas extensionales y posiblemente fracturas de cizalla que pudieran presentar cierta apertura. Consideramos que la inclusión de distintos modos de fracturas en el tratamiento de datos de apertura, más que un problema es una oportunidad para estudiar en conjunto al grupo de fracturas que se presentan en una zona de falla (Fossen, 2010). Adicionalmente, se midió la orientación e inclinación de las fracturas en cada afloramiento con el fin de establecer las diferentes familias y compararlas con los sistemas de fallas reportados en la cuenca de México. Finalmente, se realizó una descripción detallada de la geometría de las fracturas y del tipo de roca que afectan. Esto último debido a la variabilidad litológica que presentan las rocas del Mioceno en la cuenca de México (lavas, depósitos piroclásticos y volcaniclásticos). Nuestro estudio no considera a las fracturas de enfriamiento en las lavas del Mioceno, sólo a las fracturas asociadas a los tres sistemas de fallas reportados para la cuenca de México.

Sumado al trabajo de campo, se realizó un mapa detallado de lineamientos, tomando en cuenta la morfología de la parte centro-sur de la cuenca de México y considerando la metodología propuesta por Marshak y Mitra (1988) para la definición de lineamientos morfológicos usando imágenes de satélite y modelos de elevación digital. Esta actividad tuvo como propósito observar la orientación y densidad de los lineamientos en los tres sitios estudiados, y comparar su patrón (orientación y densidad) con los patrones observados en las sierras de las Cruces, Chichinautzin y Nevada.

\section{Análisis fractal}

Las fracturas son estructuras que cumplen con una característica fundamental de los objetos fractales: la invariancia en la escala, es decir, que todas sus características geométricas (longitud, apertura, orientación) se repiten en varias escalas (Mandelbrot, 1967). Además de la invariancia en la escala, los objetos fractales se caracterizan por una ley de potencia en la relación cantidad-tamaño (Barton y LaPointe, 1995; Cladouhos y Marrett, 1996; Marrett et al., 1999). En los últimos años se ha demostrado que el espaciamiento entre las fracturas y la apertura, son variables que también se pueden analizar utilizando las técnicas de la teoría fractal, ya que siguen una ley de potencia entre su cantidad y su tamaño (Hooker et al., 2013, 2018; Laubach et al., 2018; Vásquez-Serrano et al., 2019). Esto último se puede demostrar usando gráficos bilogarítmico del espaciamiento y apertura contra su frecuencia acumulada (Barton y LaPointe, 1995).

En este trabajo se utilizan herramientas de la teoría fractal para analizar el arreglo que tienen las fracturas expuestas en Sierra de Guadalupe, Tepoztlán y Malinalco. Los parámetros fractales que se usan para dicho propósito son: dimensión fractal de caja (Dc), correlación (v) y exponente de Lyapunov (Lv). La dimensión fractal de caja y correlación están relacionadas con la cantidad de fracturas y con la manera en como estas ocupan el espacio que las contienen (Barton y LaPointe, 1995; Riley et al., 2011; Vásquez-Serrano et al., 2019). En el caso del exponente de Lyapunov es importante señalar que su valor depende del grado de agrupamiento de las fracturas, es decir, si $\mathrm{Lv}=0$ se tendrán espaciamientos iguales entre las fracturas, mientras que si Lv>0 existirán grupos de fracturas (Vásquez-Serrano et al., 2019). Adicionalmente se calcula la intensidad de fracturas (I) y el coeficiente de variación $(\mathrm{Cv})$. Éste último parámetro está relacionado con la desviación estándar y el promedio de los espaciamientos; si el valor de $\mathrm{Cv}=0$ la distancia entre fracturas es igual, y cuando $\mathrm{Cv}>0$ las fracturas serán aleatorias o se agruparán (Vásquez-Serrano, 2013).

Todos estos parámetros fueron obtenidos siguiendo la metodología 
descrita por Vásquez-Serrano (2013) y Vásquez-Serrano et al. (2019) tomando como base la teoría propuesta por Cox y Lewis (1966), Davy et al. (1990), Rosenstein et al. (1993), Barton y LaPointe (1995) y Riley et al. (2011). El valor de dichos parámetros fue calculado mediante el código computacional Frac1Dv1.1 escrito en MATLAB por VásquezSerrano (2013).

\section{Suma de correlación normalizada (SCN)}

El método de SCN propuesto por Marrett et al. (2018) toma en cuenta una secuencia de espaciamientos para conocer el arreglo que tienen las fracturas en una línea de muestreo. Este método es implementado en un código computacional abierto llamado CorrCount escrito en MATLAB (Marrett et al., 2018), el cual proporciona soluciones analíticas y de Monte Carlo para datos de espaciamiento entre fracturas con un $95 \%$ de confiabilidad. El método SCN provee un análisis cuantitativo del grado de agrupamiento de las fracturas y puede ayudar a distinguir entre fracturas con un espaciamiento constante, aleatorio, o agrupadas caóticamente (de manera fractal) sin tomar en cuenta la apertura de las fracturas. El método SCN está basado en la función de correlación entre dos puntos (Bonnet et al., 2001), la cual calcula la proporción de pares de fracturas en un grupo determinado que están separados por una distancia menor a la longitud de la escala de análisis. En el método SCN, la discriminación entre los distintos arreglos de fracturas se realiza mediante patrones de las curvas observadas en los gráficos de correlación espacial de las fracturas contra la longitud de la línea de muestreo (Figura 3). Por ejemplo, para un arreglo de fracturas donde existe un agrupamiento de estas estructuras, se tendrá la formación de una pendiente negativa antes de llegar a una estabilización
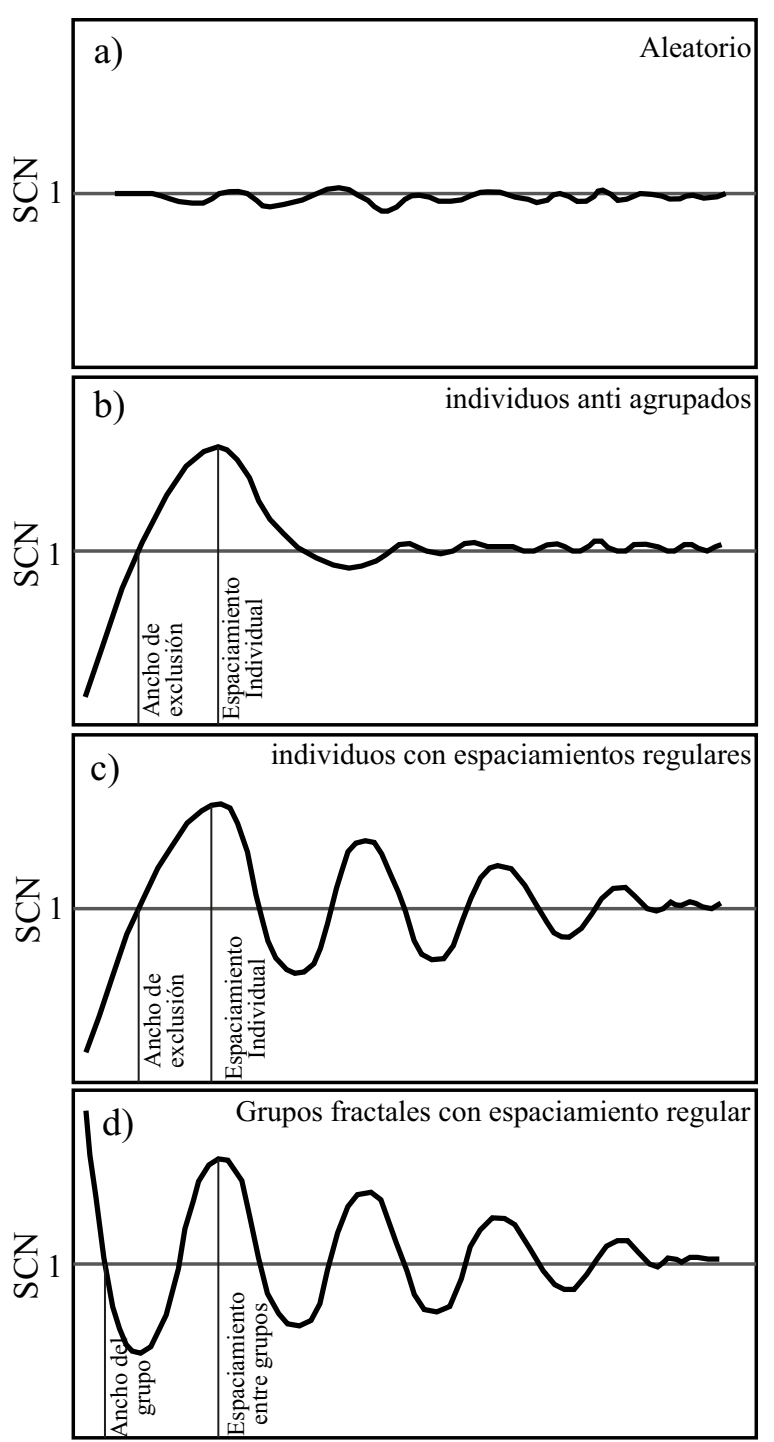

Longitud de escala (lineal)
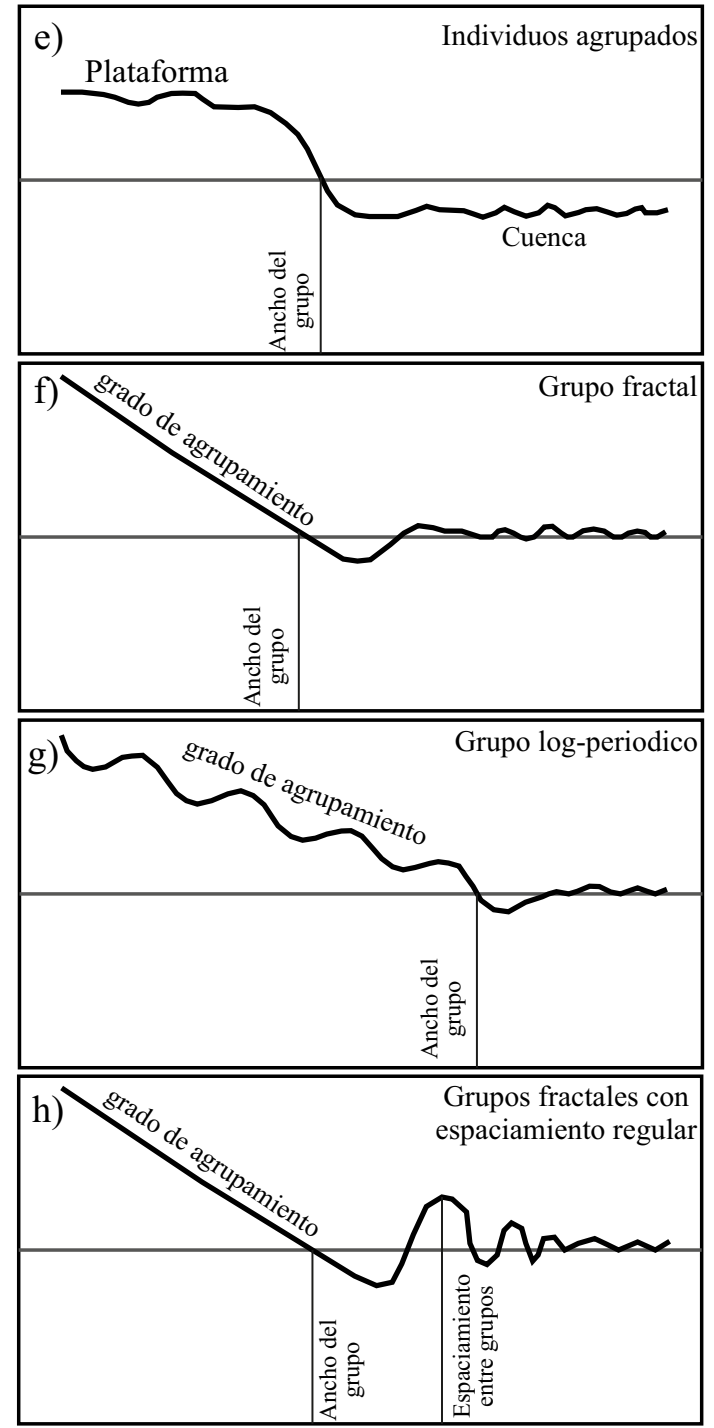

Longitud de escala (logarítmica)

* SCN (Suma de Correlación Normalizada)

Figura 3. Patrones de la variación de suma de correlación normalizada $(\mathrm{SCN})$ con la escala en formato lineal y logarítmico. a) La formación de una forma plana indica que no existe una organización estadísticamente significativa. b) Fracturas no agrupadas, pero sin espaciamiento constante. c, d, h) Patrón con altos y bajos que se relaciona con espaciamiento regular (igual espaciamiento). f, g, h) Variación asociada a una ley de potencia en el agrupamiento de fracturas con la escala (comportamiento fractal), dicho patrón se observa mejor en el eje logarítmico de la escala. e) Formación de plataforma y cuenca que indica agrupamiento de fracturas relacionadas con procesos de herencia estructural y/o reactivación. Modificado de Marrett et al. (2018). 
de la curva que se dibuja (Figura 3). Una característica importante del método SCN, es que puede ser aplicado a cualquier modo de fracturas debido a que no toma en cuenta la apertura (Marrett et al., 2018)

Para el estudio presentado aquí, la obtención de los datos de espaciamiento para el análisis de SCN tomó en cuenta a todas las fracturas subverticales y verticales observadas en afloramientos con más de $500 \mathrm{~m}^{2}$, donde se obtuvieron líneas de muestreo de entre 40 y $200 \mathrm{~m}$ de longitud. El análisis de SCN también toma en cuenta a dos líneas de muestreo combinadas (que incluyen a más de una familia de fracturas), donde los espaciamientos son aparentes. El tamaño de estas líneas de muestreo, permitió obtener una cantidad de datos suficientes para analizar el arreglo de las fracturas utilizando la técnica del SCN.

\section{RESULTADOS}

Fracturas que afectan a las rocas miocénicas de la cuenca de México Las fracturas que afectan a las rocas del Mioceno dentro (Sierra de Guadalupe) y en los bordes de la Cuenca de México (Malinalco y Tepoztlán) son principalmente híbridas, aunque también existen fracturas extensionales (Modo I) y de cizalla (Modo II y III) (Figura 4). Todas estas fracturas están asociadas con fallas que tienen una cinemática normal y lateral izquierda (Figura 4a), y son paralelas al rumbo de dichas fallas. Las fracturas híbridas y de cizalla se localizan principalmente en la zona de afectación de las fallas, no tienen evidencia de relleno y presentan paredes pintadas de óxidos. La presencia de estas fracturas suele extenderse hasta por $200 \mathrm{~m}$ a partir del plano de falla en algunos sitios, como se observó en la zona de Tepoztlán, Morelos. La apertura de las fracturas híbridas es menor a $2 \mathrm{~cm}$ en promedio, presentando la mayor apertura las fracturas localizadas cerca del núcleo de las fallas. En el caso de las fracturas extensionales, éstas se encuentran en muchos casos abiertas con oxidación en sus paredes, lo cual evidencia el paso de fluidos a través de ellas. En zonas cercanas a las fallas kilométricas, este tipo de fracturas también se encuentran rellenas de arcilla color verde y marrón. La apertura de este conjunto de fracturas va desde $1 \mathrm{~mm}$ hasta poco menos de $1 \mathrm{~m}$.

Con base en su orientación, las fracturas se dividieron en 3 familias (grupos de fracturas paralelas) sin importar su cinemática (Modo I, II, III o híbridas). La familia F1 está representada por fracturas subverticales que tienen un rumbo NE-SW, las cuales están asociadas a fallas laterales con cinemática izquierda y afectan principalmente a las rocas de la Sierra de Guadalupe, aunque también se observaron en el área de Tepoztlán y de manera escasa en Malinalco (Figura 4a y $4 b)$. Por otra parte, la familia F2 está constituida por fracturas subverticales con rumbo NNW-SSE, relacionadas con un sistema de fallas normales con rumbo preferencial N-S que cortan a las fallas laterales izquierdas en la Sierra de Guadalupe (Figura $4 \mathrm{a}$ y $4 \mathrm{~b}$ ). Las fracturas F2 afectan a las rocas miocénicas de los tres sitios estudiados y son las que más frecuentemente se observan en los afloramientos. Finalmente, la familia F3 está conformada por fracturas subverticales con rumbo preferencial $\sim \mathrm{E}-\mathrm{W}$, paralelas al rumbo de fallas kilométricas de tipo normal dentro de la cuenca de México (ej. Falla Xochimilco). Al igual que la familia F2, la familia F3 se presenta en los tres sitios estudiados. Tanto a escala de afloramiento como a escala de un mapa, se observa que las fracturas de la familia F3 cortan a las familias F1 y F2, lo que sugiere que este grupo de fracturas es el más joven dentro de la cuenca de México (Figura 4a y 4b).

\section{Distribución espacial de las fracturas}

El análisis morfológico de lineamientos de la parte centro-sur de la cuenca de México revela que en los sitios donde se exponen rocas del Mioceno (Sierra de Guadalupe, Malinalco y Tepoztlán), se encuentra la mayor densidad de lineamientos en comparación con otros sitios donde afloran rocas más jóvenes (Sierra de las Cruces, Sierra Nevada y Sierra Chichinautzin) (Figura 4c). La orientación de dichos lineamientos permite agruparlos en tres familias con un rumbo preferencial NE-SW, NNW-SSE y E-W (Figura 4 b). El rumbo principal de los lineamientos es congruente con la orientación de los principales sistemas de fallas reportados dentro de la cuenca de México.

A escala de afloramiento, en cada sitio estudiado (Sierra de Guadalupe, Malinalco y Tepoztlán) se observó la presencia de tres familias de fracturas (F1, F2 y F3) subparalelas a los lineamientos morfológicos a escala kilométrica (Figura 4). La distribución espacial de las fracturas en las líneas de muestreo, revela que cada familia de fracturas tiende a formar grupos, sobre todo en aquellos lugares donde hay presencia de fallas (Figura 5). Este comportamiento en la distribución espacial de las fracturas parece ser independiente del tipo de roca. Tanto en depósitos volcaniclásticos como en paquetes de lavas predomina la tendencia a formar grupos de fracturas observables en las líneas de muestreo de diferente longitud (Figura 5). En las líneas de muestreo que incluyen a dos familias de fracturas (líneas de muestreo combinadas) es posible observar de manera cualitativa un incremento en el grado de agrupamiento (Figura $5 \mathrm{~d}$ ), contrario a lo que se observa en líneas que incluyen a una sola familia (Figura 5a, 5b, 5c).

Exponente de frecuencia acumulada de espaciamiento y apertura

Una manera de analizar la abundancia de la apertura y el espaciamiento un rango de escala dado es mediante un gráfico bilogarítmico frecuencia acumulada contra el tamaño, donde la pendiente (exponente de frecuencia acumulada) mostrará la proporción cantidad-tamaño de estas variables. Tomando en cuenta todos los datos de apertura colectados en los tres sitios de estudio (Sierra de Guadalupe, Malinalco y Tepoztlán), ésta variable muestra un comportamiento fractal, ya que sigue una ley de potencia. Las fracturas F1 tienen el exponente de frecuencia acumulada más alto con un valor absoluto de 5.061, mientras que las fracturas F2 presentan el valor más bajo con 1.692. Para las fracturas F3 se obtuvo un exponente de frecuencia acumulada de 2.227 (Figura 6). Aunque el exponente de frecuencia acumulada para F1 tiene un valor alto en comparación con otros datos de apertura reportados (Hooker et al., 2013), un aspecto que hay que tomar en cuenta es que nuestro estudio toma en cuenta no sólo a las fracturas extensionales, sino que incluye a los otros modos de fracturas, lo cual puede explicar el valor anómalo de F1. El espaciamiento en las tres familias de fracturas también tiene un comportamiento fractal, demostrado por la línea recta en la pendiente que se dibuja en los gráficos de frecuencia acumulada contra espaciamiento de la Figura 7 (función de ley de potencia). En los tres sitios estudiados, las fracturas F2 presentan los valores más altos en el exponente de frecuencia acumulada (2.51 a 4.19), mientras que las fracturas F1 y F3 tienen valores similares (1.81 a 2.59).

\section{Parámetros fractales}

El espaciamiento entre fracturas medido en una línea de muestreo (Figura 5) es susceptible de analizarse mediante el cálculo de parámetros fractales como: exponente de Lyapunov, coeficiente de variación, dimensión fractal de caja y correlación. Estos parámetros suelen utilizarse para extraer información valiosa de la distribución espacial y arreglo que tienen las fracturas en una dimensión (Vásquez-Serrano et al., 2019). Las dimensiones fractales de caja y correlación se relacionan con la cantidad y la distribución espacial de las fracturas (Barton y LaPointe, 1995; Vásquez-Serrano, 2013). Si el valor de estos parámetros es cercano a 1 , se tendrá una cantidad suficientemente grande de fracturas que cuando sean intersectadas por una línea de muestreo, éstas son capaces de cubrir casi en su totalidad a dicha línea. También puede conseguirse el valor igual a 1 cuando las fracturas esta repartidas de manera homogénea en el espacio (Vásquez-Serrano, 2013). 


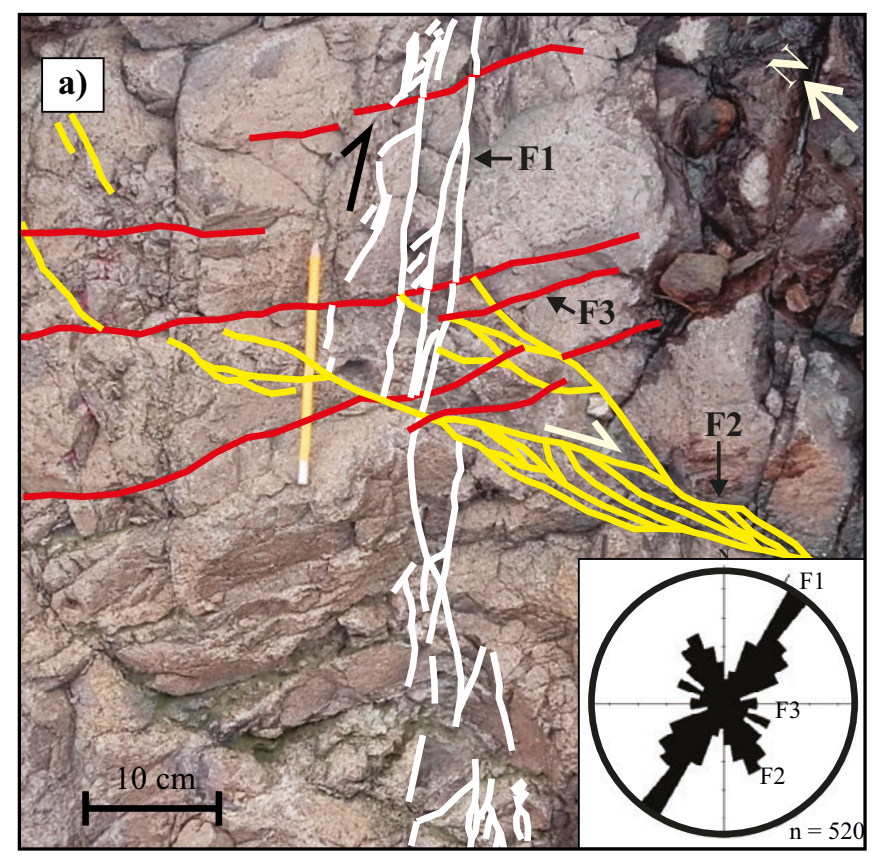

b) Lineamientos en rocas del Mioceno
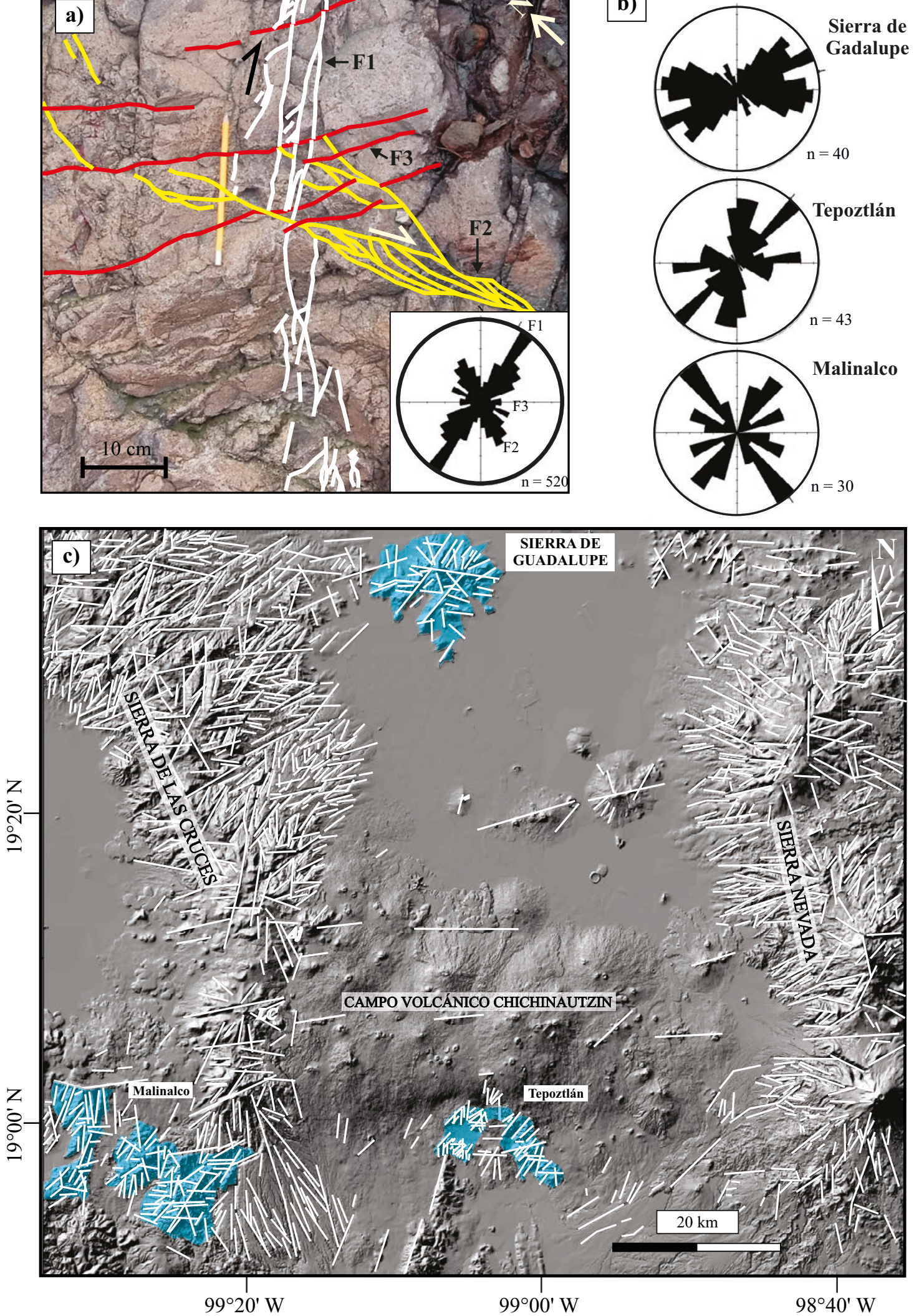

Figura 4. a) Datos estructurales de fracturas medidos en campo (diagrama de rosa) y familias de fracturas (F1, F2 y F3) que afectan a las rocas del Mioceno de la cuenca de México (Fotografía de un afloramiento de la Sierra de Guadalupe). b) Diagrama de rosa de lineamientos morfológicos de los sitios en donde se exponen rocas del Mioceno dentro (Sierra de Guadalupe) y en los alrededores (Malinalco y Tepoztlán) de la cuenca de México. c) Mapa de lineamientos morfológicos en la cuenca de México, elaborado con base en la metodología propuesta por Marshak y Mitra (1988). 


\section{Distribución de fracturas en líneas de muestreo}

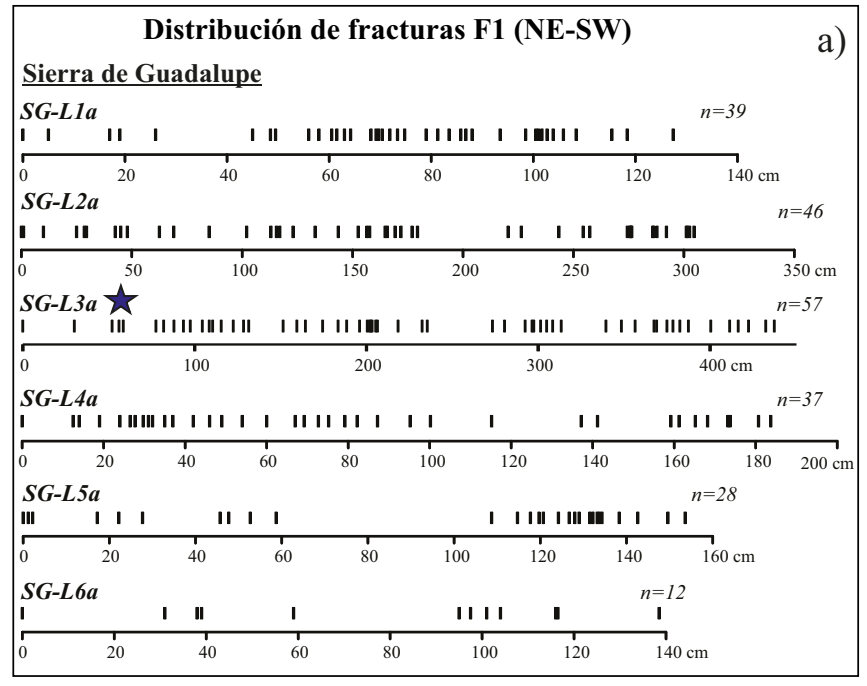

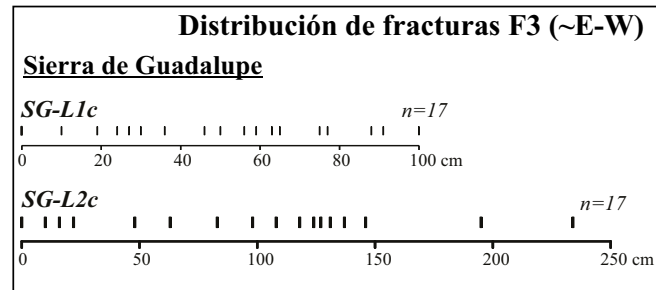

Malinalco

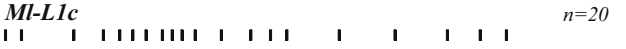

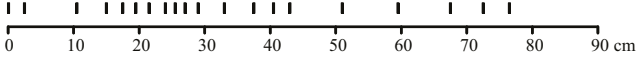

Ml-L2c

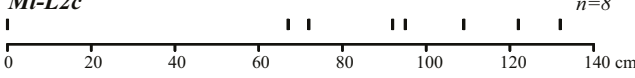

MI-L3c $n=10$

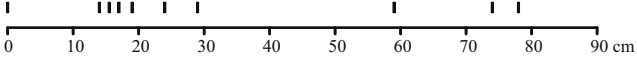

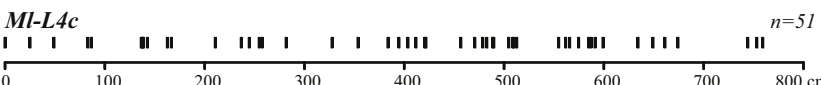

MI-L5c , । । । । । । $n=32$

\begin{tabular}{llllcl}
\hline 0 & 50 & 100 & 150 & 200 & $250 \mathrm{~cm}$
\end{tabular}

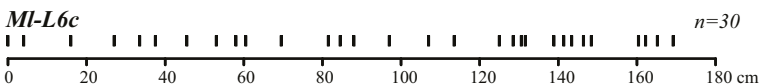

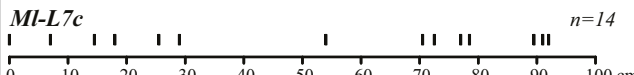

Tepoztlán

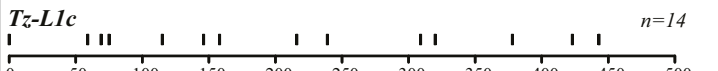

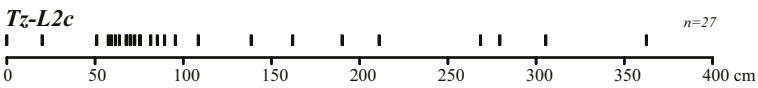

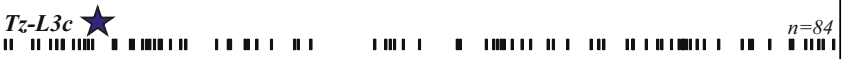

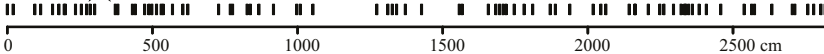

c)
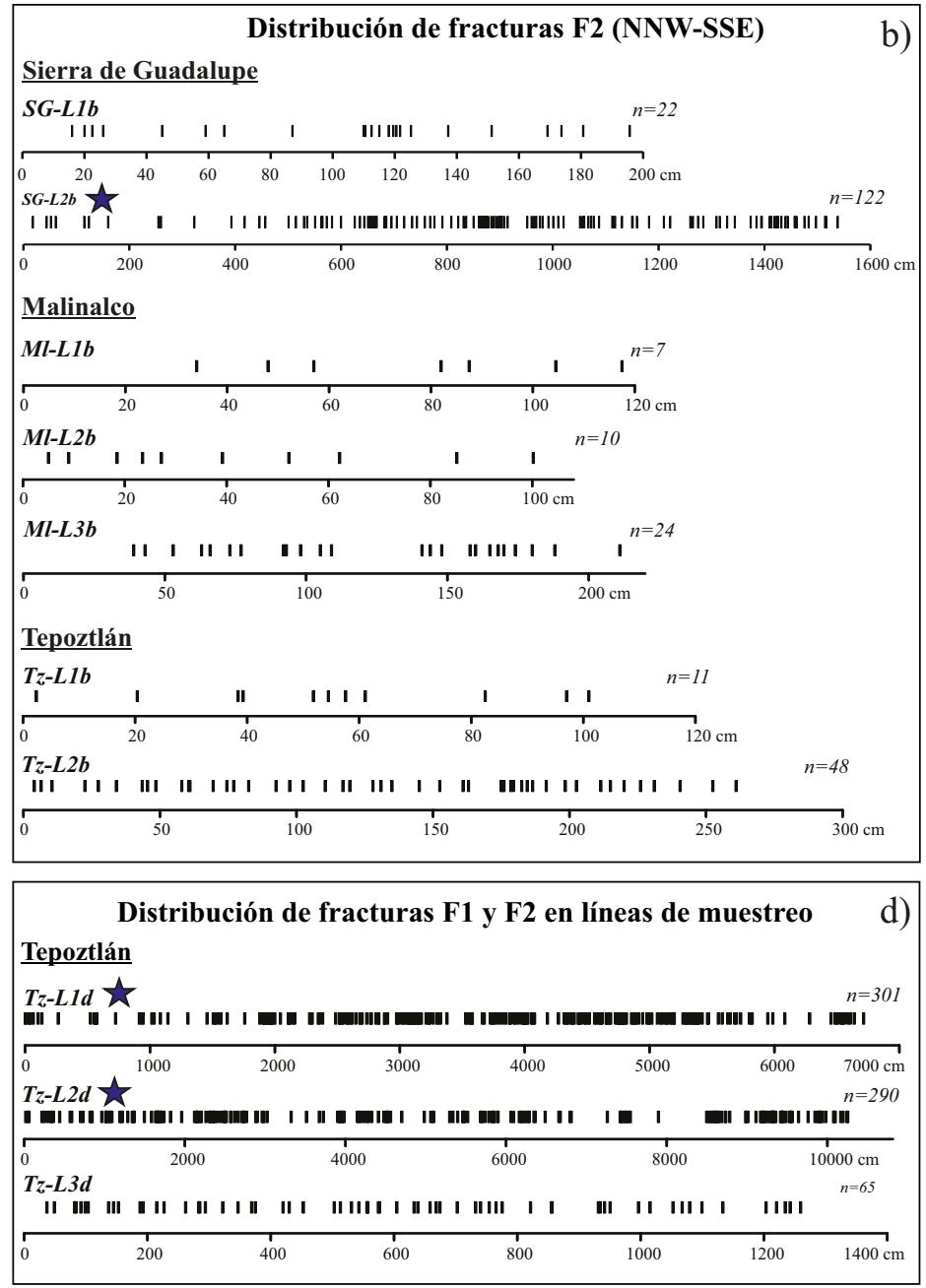

Figura 5. a, b, c) Distribución de fracturas en líneas de muestreo para las tres familias estudiadas (F1, F2 y F3). d) Distribución de tres líneas de muestreo medidas en Tepoztlán que involucra a dos familias de fracturas (F1 y F2). Las estrellas azules indican las líneas de muestreo que se usaron para el análisis de Suma de Correlación Normalizada SCN. 

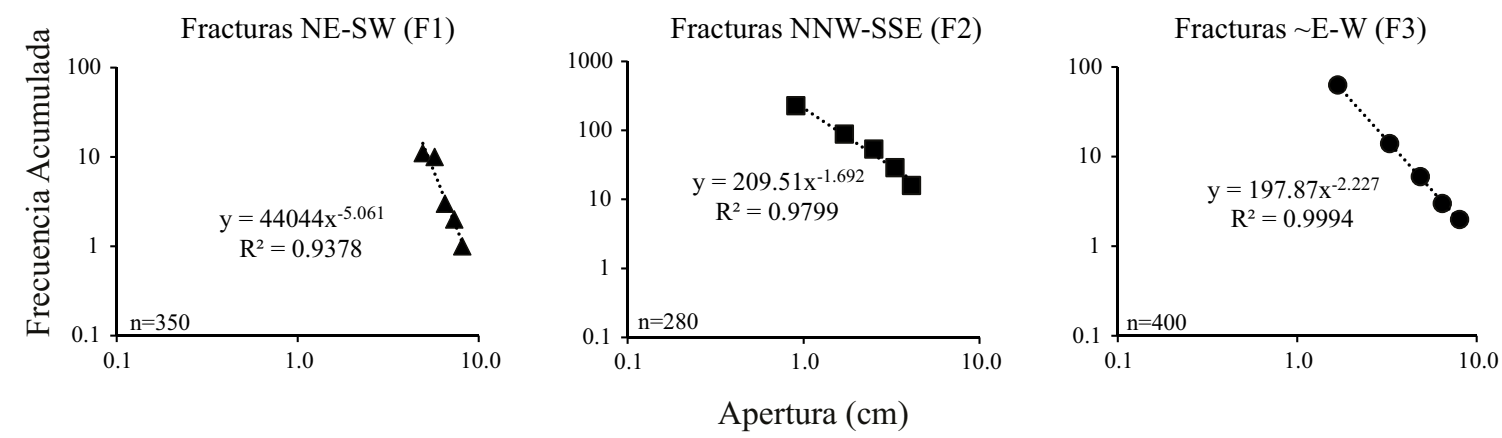

Figura 6. Gráfico bilogarítmico del tamaño de la apertura contra su frecuencia acumulada para las fracturas F1, F2 y F3.

Contrariamente si existe una cantidad pequeña de fracturas que son contadas por una línea de muestreo y las fracturas ocupan posiciones tales que forman grupos, el valor de las dimensiones fractales se aproximará a cero (Vásquez-Serrano, 2013). Para el caso de las familias de fracturas que afectan a las rocas del Mioceno en la cuenca de México, las dimensiones fractales de caja y correlación tienen valores entre 0.9 y 0.3 (Tabla 1). Este amplio rango de valores es un reflejo de la distribución espacial y cantidad de fracturas cortadas en las líneas de muestreo (Figura 5).

El exponente de Lyapunov es un parámetro que se correlaciona con el arreglo que tienen las fracturas. Un arreglo con fracturas agrupadas tendrá valores de este parámetro mayores a cero (Riley et al., 2011). Con un agrupamiento de fracturas cada vez más marcado aumentará el valor del exponente de Lyapunov (Vásquez-Serrano, 2013). En las rocas miocénicas de la cuenca de México, las fracturas presentan valores del exponente de Lyapunov mayores a 1 en su gran mayoría, sólo en algunas líneas de muestreo se obtuvieron valores en un rango de 0.53-0.93. Un aspecto relevante es que las líneas de muestreo que incluyen a dos familias de fracturas (F1 y F2) en Tepoztlán, presentan valores más o menos homogéneos en un rango de 1.56 a 1.67 (Tabla 1).
Sierra de Guadalupe
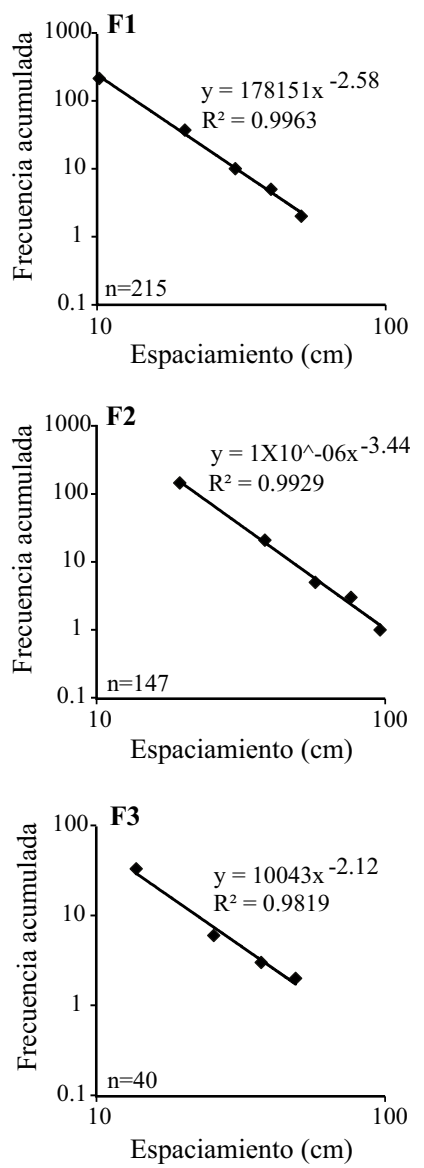

\section{Tepoztlán}
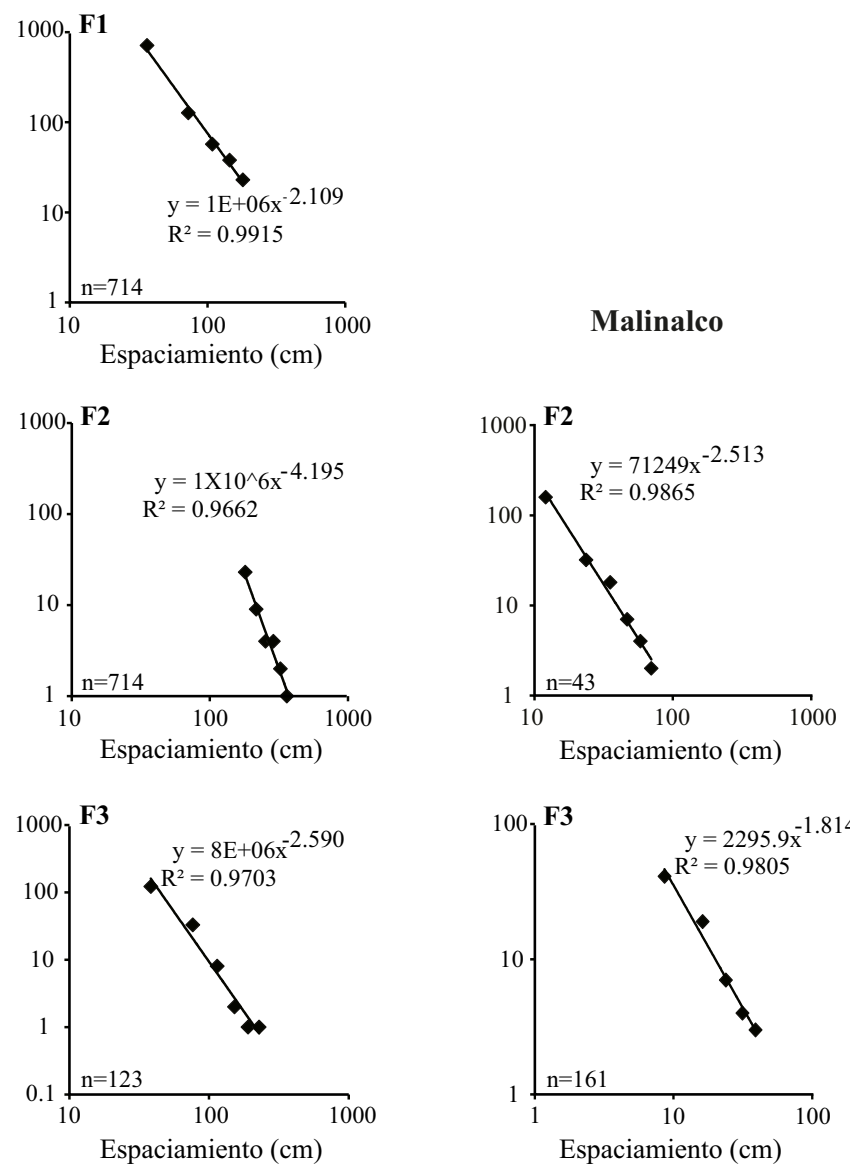

Malinalco

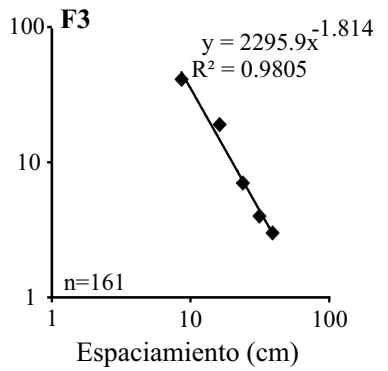

Figura 7. Exponentes de frecuencia acumulada del espaciamiento para los tres sitios estudiados. En Sierra de Guadalupe y Malinalco se obtuvieron datos para las fracturas F1, F2 y F3 mientras que para Tepoztlán sólo se midieron espaciamientos entre fracturas F2 y F3. 
Tabla 1. Valores de los parámetros fractales en cada línea de muestreo.

\begin{tabular}{|c|c|c|c|c|c|c|c|}
\hline & & $\begin{array}{c}\text { Dimensión } \\
\text { de caja }\end{array}$ & $\begin{array}{l}\text { Dimensión } \\
\text { de correlación }\end{array}$ & $\begin{array}{l}\text { Exponente } \\
\text { de Lyapunov }\end{array}$ & $\begin{array}{l}\text { Coeficiente } \\
\text { de variación }\end{array}$ & Intensidad & Tipo de roca \\
\hline \multicolumn{8}{|c|}{ Sierra de Guadalupe } \\
\hline \multirow[t]{6}{*}{ F1: } & $S G-L 1 a$ & 0.71 & 0.42 & 1.08 & 1.09 & 30.61 & Lava \\
\hline & $S G-L 2 a$ & 0.77 & 0.45 & 1.07 & 1.10 & 15.10 & Lava \\
\hline & $S G-L 3 a$ & 0.80 & 0.49 & 1.13 & 0.95 & 13.03 & Lava \\
\hline & $S G-L 4 a$ & 0.75 & 0.42 & 1.12 & 0.93 & 20.14 & Lava \\
\hline & $S G-L 5 a$ & 0.64 & 0.28 & 1.26 & 1.07 & 18.22 & Lava \\
\hline & $S G-L 6 a$ & 0.36 & 0.42 & 1.42 & 1.01 & 8.66 & Lava \\
\hline \multirow[t]{2}{*}{$F 2$ : } & $S G-L 1 b$ & 0.57 & 0.44 & 1.17 & 0.88 & 12.26 & Lava \\
\hline & $S G-L 2 b$ & 0.88 & 0.50 & 1.21 & 1.11 & 7.99 & Depósitos volcaniclásticos \\
\hline \multirow[t]{2}{*}{ F3: } & $S G-L 1 c$ & 0.57 & 0.49 & 1.08 & 0.55 & 18.00 & Lava \\
\hline & $S G-L 2 c$ & 0.54 & 0.48 & 0.70 & 0.90 & 27.26 & Lava \\
\hline \multicolumn{8}{|l|}{ Malinalco } \\
\hline \multirow[t]{3}{*}{$F 2$ : } & $M l-L 1 b$ & 0.64 & 0.53 & 1.43 & 1.09 & 6.80 & Depósitos volcaniclásticos \\
\hline & $M l-L 2 b$ & 0.38 & 0.46 & 0.93 & 0.61 & 10.97 & Lava \\
\hline & $M l-L 3 b$ & 0.27 & 0.43 & 1.08 & 0.58 & 11.84 & Lava \\
\hline & $M l-L 1 c$ & 0.62 & 0.48 & 0.53 & 0.60 & 26.14 & Lava \\
\hline & $M l-L 2 c$ & 0.36 & 0.37 & 1.60 & 1.17 & 6.06 & Depósitos volcaniclásticos \\
\hline & $M l-L 3 c$ & 0.34 & 0.32 & 1.16 & 1.09 & 12.82 & Lava \\
\hline & $M l-L 4 c$ & 0.77 & 0.58 & 1.66 & 1.39 & 6.71 & Depósitos volcaniclásticos \\
\hline & $M l-L 5 c$ & 0.68 & 0.28 & 1.37 & 1.02 & 15.45 & Lava \\
\hline & $M l-L 6 c$ & 0.73 & 0.61 & 1.01 & 0.62 & 17.73 & Lava \\
\hline & $M l-L 7 c$ & 0.45 & 0.48 & 1.46 & 0.98 & 15.21 & Lava \\
\hline \multicolumn{8}{|l|}{ Tepoztlán } \\
\hline \multirow[t]{2}{*}{ F2: } & $T z-L 1 b$ & 0.38 & 0.30 & 1.17 & 0.84 & 11.88 & Depósitos volcaniclásticos \\
\hline & $T z-L 2 b$ & 0.85 & 0.73 & 1.12 & 0.60 & 18.77 & Depósitos volcaniclásticos \\
\hline \multirow[t]{3}{*}{ F3: } & $T z-L 1 c$ & 0.44 & 0.48 & 1.51 & 1.65 & 3.16 & Depósitos volcaniclásticos \\
\hline & $T z-L 2 c$ & 0.55 & 0.46 & 1.38 & 0.65 & 7.44 & Depósitos volcaniclásticos \\
\hline & $T z-L 3 c$ & 0.87 & 0.50 & 1.42 & 0.95 & 2.94 & Depósitos volcaniclásticos \\
\hline \multicolumn{8}{|c|}{ Combinado (Tepoztlán) } \\
\hline \multirow[t]{3}{*}{$F 1+F 2$} & $T z-L 1 d$ & 0.90 & 0.40 & 1.56 & 1.58 & 4.48 & Depósitos volcaniclásticos \\
\hline & $T z-L 2 d$ & 0.88 & 0.30 & 1.67 & 1.84 & 2.82 & Depósitos volcaniclásticos \\
\hline & $T z-L 3 d$ & 0.86 & 0.80 & 1.64 & 1.82 & 5.24 & Depósitos volcaniclásticos \\
\hline
\end{tabular}

Los coeficientes de variación obtenidos están entre $0.58-1.82$, lo que sugieren que las fracturas tienden a agruparse. En las líneas de muestreo que incluyen a dos familias de fracturas (F1 y F2) se observa el coeficiente de variación más alto, con un rango de entre 1.58 y 1.82 (Tabla 1).

Además de los parámetros fractales antes descritos, también se calculó la intensidad de fractura (número de fracturas por unidad de longitud) en cada línea de muestreo. En general, la intensidad de fracturas varía entre 2.82 y 30.61 fracturas/m en las tres familias de fracturas (F1, F2 y F3). El valor de la intensidad tiene una relación muy clara con el tipo de roca, siendo las lavas (andesita) las que presentan los mayores valores (8.66 a 30.61) y los depósitos volcaniclásticos los que tienen los valores más bajos (2.82-7.99). Es importante mencionar la relación inversa que guarda la intensidad de fracturas con el exponente de Lyapunov, como se observa en la Tabla 1; cuando la intensidad es baja el valor de este parámetro es alto.

\section{Caracterización de grupos de fracturas}

Para analizar de manera más detallada a los grupos de fracturas, se procedió a graficar la suma de correlación normalizada (SCN) en un gráfico bilogarítmico contra la escala (Figura 8). En este mismo gráfico se incluye la variación de la intensidad de fracturas (normalizada) a lo largo de las líneas de muestreo seleccionadas para el análisis (Figura 8). Si se compara el patrón obtenido en la SCN para cada familia de fracturas (F1, F2 y F3) con lo que se observa en la Figura 3, es posible distinguir ciertas características en el arreglo de los grupos de fracturas de cada familia. Las fracturas F1 muestran la formación de una pendiente en el grafico bilogarítmico de la Figura 8b, lo que sugiere que la distribución espacial de los grupos se comportan como un fractal, es decir, son autosimilares y la relación frecuencia-tamaño sigue una ley de potencia. El tamaño máximo de los grupos de fracturas F1 es de aproximadamente $80 \mathrm{~cm}$ en por lo menos dos órdenes de escala (Figura 8b). En el caso de las fracturas F2, éstas generan un patrón de meseta que se aleja de una tendencia aleatoria. Esta meseta (plataforma) abarca por lo menos dos órdenes de escala $\left(10^{1}-10^{3}\right)$ con un tamaño máximo de los grupos de $110 \mathrm{~cm}$ (Figura 8d). Para ambas familias de fracturas (F1 y F2) se observa la formación de picos pronunciados en los gráficos de intensidad de fracturas normalizada, lo cual es congruente con la formación de grupos de fracturas muy marcados en las líneas de muestreo. Finalmente, las fracturas F3 se ajustan a una distribución espacial aleatoria de los grupos de fracturas dentro del rango de escala estudiado (Figura 8f), esto es corroborado en el gráfico de intensidad de fracturas normalizada donde no se observa ningún pico importante (Figura 8e). 


\section{DISCUSIÓN}

Variaciones litológicas y su relación con el arreglo de fracturas.

Las rocas del Mioceno dentro de la cuenca de México están representadas por una intercalación de lavas (andesitas, dacitas) y depósitos volcaniclásticos, lo cual imparte al paquete de roca una reología muy heterogénea (Arce et al., 2019). En los tres sitios estudiados es posible observar dicha variación litológica, siendo la Sierra de Guadalupe la que está representada mayoritariamente por lavas y la zona de Tepoztlán la que tiene en su mayoría depósitos volcaniclásticos. Pozos profundos realizados por PEMEX y el Sistema de Aguas de la Ciudad de México en el siglo pasado y en la última década, han cortado una sucesión de rocas del Mioceno dentro de la Ciudad de México a profundidades de entre $700 \mathrm{~m}$ y hasta más de $1500 \mathrm{~m}$, que demuestran que la variación litológica que se observa en superficie tiene continuidad a profundidad (Arce et al., 2013, 2015, 2019). La heterogeneidad litológica es una variable fundamental que influye en el estilo y cantidad de deformación durante un evento tectónico (Fitz-Díaz et al., 2012; Vásquez-Serrano et al., 2018a).

Las diferentes familias de fracturas que afectan a las rocas del Mioceno en la cuenca de México están subordinadas al tipo de material que afectan. Las lavas suelen contener un mayor número de fracturas, reflejado en una alta intensidad, mientras que los depósitos volcaniclásticos, contrariamente presentan una menor intensidad de

Fracturas F1(NE-SW)
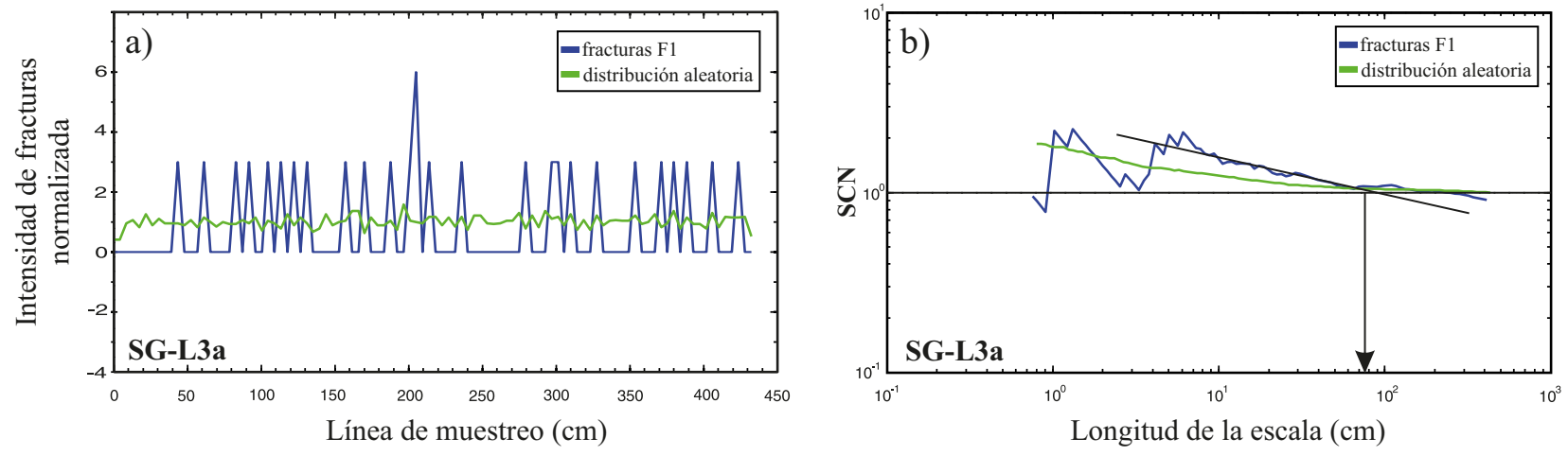

Fracturas F2 (NNW-SSE)
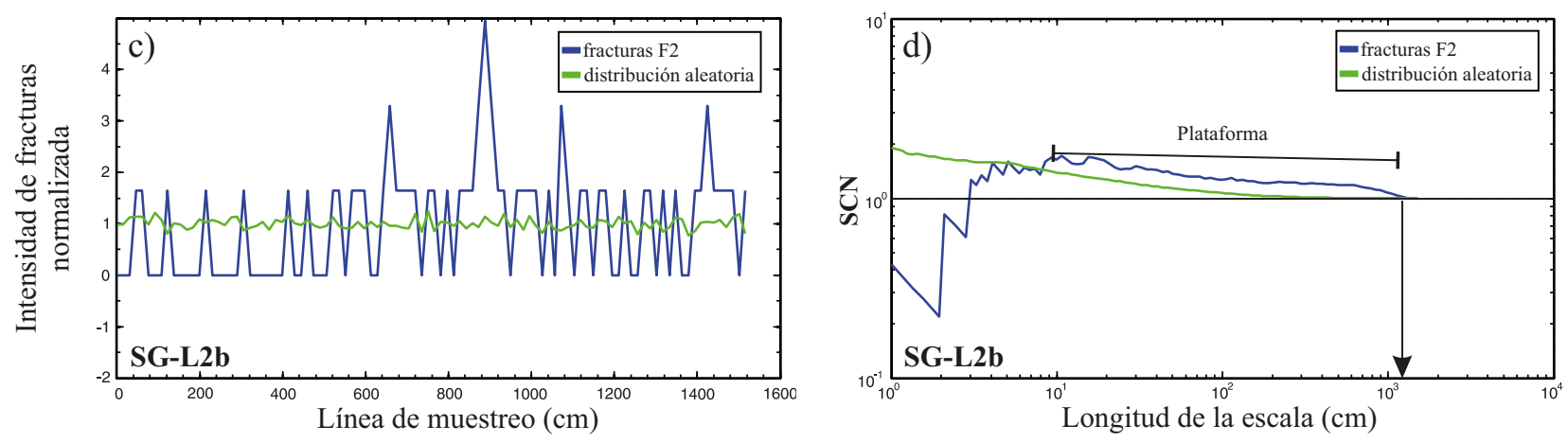

Fracturas F3(E-W)
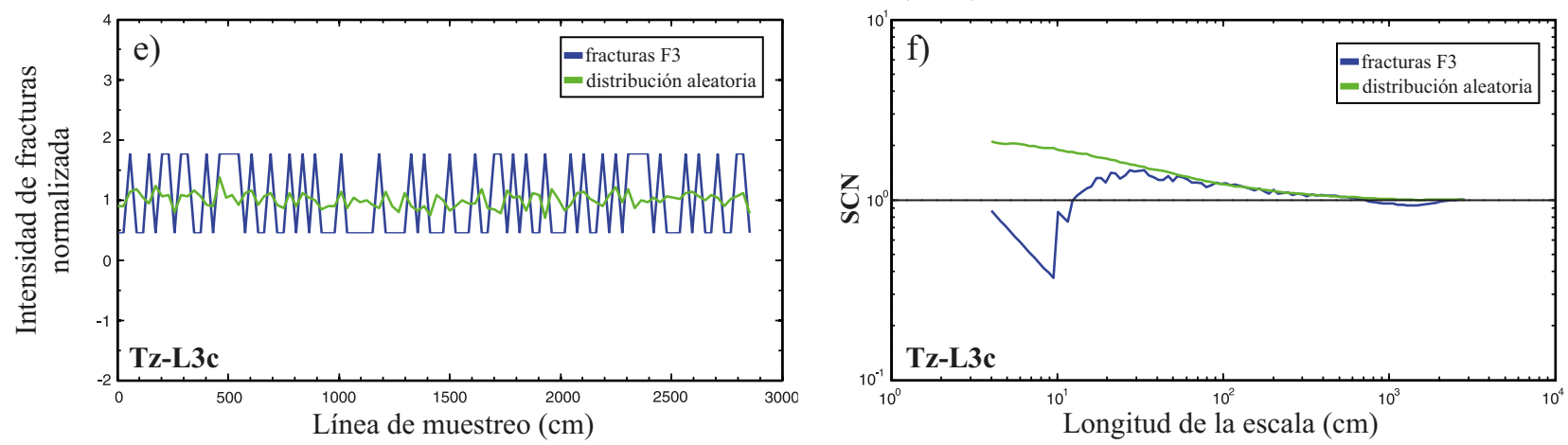

Figura 8. a, c, e) Gráficos de intensidad de fracturas normalizado y (b, d, f) suma de correlación normalizada (SCN) para las familias de fracturas estudiadas (F1, F2 y F3). Las curvas obtenidas de los datos de espaciamiento (línea azul) son comparadas con una curva de referencia asociada a fracturas con la formación de grupos con una distribución aleatoria (línea verde). El análisis fue realizado en las líneas de muestreo que tienen la cantidad mayor de fracturas en cada familia de los tres sitios (ver figura 5). a, b) Las fracturas F1 muestran una distribución de grupos de fracturas que tiene una distribución fractal. c, d) Para las fracturas F2 se observa la formación de una plataforma, relacionada con grupos de fracturas ligados a anisotropías en rocas pre-miocénicas. e, f) En cuanto a las fracturas F3, la curva de SCN se ajusta a un patrón aleatorio en la distribución de los grupos de fracturas. 
fracturas (Tabla 1). Es relevante el arreglo que tienen las fracturas en cada tipo de roca, el análisis realizado en este trabajo sugiere que las lavas tienden a formar arreglos menos agrupados en comparación con los depósitos volcaniclásticos. Este patrón es soportado con los valores del exponente de Lyapunov que se observan en cada tipo de roca (Tabla 1). Un aspecto interesante es la variación de la intensidad de fracturas en función del grado de agrupamiento (exponente de Lyapunov), donde es posible observar que existe una relación inversa entre ambos parámetros (Figura 9). Nuestros datos sugieren que a mayor cantidad de fracturas en una roca disminuye el grado de agrupamiento en su arreglo. Este patrón contradice lo reportado en Vásquez-Serrano et al. (2019), sin embargo, consideramos que dicha contradicción puede ser debida a que en el trabajo de Vásquez-Serrano et al. (2019) se reporta una concentración anómala de fracturas (alta intensidad de fracturas) debido a que se encuentran en una zona de cizalla frágil que además afecta a fracturas previas. Esto ocasiona que aumente el grado de agrupamiento conforme aumenta la intensidad de fracturas. El patrón observado en Vásquez-Serrano et al. (2019) en una zona de falla, es similar a lo que se reporta en nuestro trabajo en sitios lejanos a la zona anómala ubicada cerca del núcleo de una falla.

En el caso de las rocas en estudio, los depósitos volcaniclásticos son afectados por fracturas que tienen un arreglo de grupos muy marcados con una intensidad de fracturas menor en comparación con las lavas. Éstas últimas tienen fracturas que presentan un arreglo menos agrupado. El patrón observado en el arreglo e intensidad de fracturas es un reflejo del acomodo de la deformación en cada material y consecuentemente influye en la generación de permeabilidad secundaria. Paquetes de lava dentro de las rocas del Mioceno tendrán una mayor cantidad de fracturas con un arreglo menos agrupado en comparación con los depósitos volcaniclásticos. Esta característica incrementa la porosidad y la permeabilidad en las lavas, siendo un paquete con gran potencial para albergar un acuífero en el subsuelo de la Ciudad de México. Será importante en el futuro hacer un estudio cuantitativo de la porosidad y permeabilidad de la lavas para conocer con exactitud su potencial como acuífero en el valle de México.

Origen, distribución y arreglo de fracturas en la cuenca de México

Las fracturas que afectan a las rocas del Mioceno en la Cuenca de México presentan valores característicos en los parámetros fractales que distinguen a cada familia. El exponente de frecuencia acumulada del espaciamiento es un parámetro que puede ayudar a distinguir una familia de fracturas de otra en un sitio determinado mediante una línea de muestreo. Nuestros resultados sugieren que aun cuando se toma en cuenta a más de una familia de fracturas en una línea de muestreo, se puede determinar qué familias se están observando. La formación de dos pendientes en un gráfico de frecuencia acumulada del espaciamiento se relaciona con un objeto multifractal y refleja la inclusión de dos familias de fracturas en una línea de muestreo (Barton y LaPointe, 1995; Vásquez-Serrano et al., 2019). En los tres sitios estudiados (Sierra de Guadalupe, Malinalco y Tepoztlán), las fracturas F2 tienen el valor más alto del exponente de frecuencia acumulada del espaciamiento (Figura 7). Esto se relaciona con la presencia de una mayor cantidad de espaciamientos pequeños (entre 10 y $50 \mathrm{~cm}$ ) en comparación con espaciamientos grandes $(>1 \mathrm{~m})$. Con referente a las fracturas F1 y F3, éstas presentan valores del exponente de frecuencia acumulada de 1.8 a 2.5, lo que sugiere que existe una mayor cantidad de espaciamientos grandes $(>80 \mathrm{~cm})$ y un menor número de espaciamientos pequeños (entre 10 y $50 \mathrm{~cm}$ ).

En el caso de las dimensiones fractales de caja y correlación, éstas no muestran una variación significativa que ayude a diferenciar cada familia de fracturas (Tabla 1). Un comportamiento similar se observa en el coeficiente de variación y el exponente de Lyapunov (Tabla 1).
Para este último parámetro se observa una relación lineal con la intensidad de fracturas, la cual está íntimamente relacionada con el tipo de roca (Figura 9).

Las dimensiones fractales tienen valores promedios de 0.63 y 0.46 para la dimensión de caja y correlación respectivamente, tomando en cuenta a todas las familias de fracturas. Estos valores sugieren que las fracturas F1, F2 y F3 ocupan entre 46 y $63 \%$ del espacio total en una línea de muestreo y que están agrupadas. Comúnmente se ha asociado la dimensión de caja con la cantidad de fracturas (densidad) que hay en una superficie (o línea de muestreo) (Nieto-Samaniego et al., 2003, 2005; Vásquez-Serrano, 2013) de un tipo de roca en particular, sin embargo, en el caso de las fracturas asociadas con fallas (Modo I, II y III) además del tipo de roca se encuentra la concentración de las fracturas (aumento en la cantidad de fracturas) en zonas de deformación localizada (zonas de falla) (Ramsay, 1967; Ramsay y Huber, 1987; Brogi, 2011; Vásquez-Serrano et al., 2019). Por otro lado, el exponente de Lyapunov y coeficiente de variación demuestran que las fracturas que afectan a las rocas miocénicas tienen un arreglo agrupado. Este arreglo presenta diferentes grados de agrupación que depende del tipo de roca (Tabla 1; Figura 9). De acuerdo con algunos trabajos (Riley et al., 2011; Odling et al., 1999; Gillespie et al., 1993; Vásquez-Serrano, 2013) el arreglo de fracturas agrupadas es común en muchos tipos de rocas. Este fenómeno ha sido investigado por algunos autores (Holder et al., 2001; Olson, 2003), quienes han sugerido que existe un control mecánico en el patrón agrupado que se observa en los sistemas de fracturas. Olson (1993) sugiere que la velocidad de propagación de la fractura, el factor de intensidad de tensiones y la tenacidad del material influyen, en gran medida, en el arreglo de las fracturas a través del índice subcrítico de fractura, el cual es diferente para cada tipo de roca.

Los patrones observados en los gráficos de suma de correlación normalizada (Figura 3), además de dar información del arreglo que tienen las fracturas, también pueden ayudar a distinguir familias de fracturas. Para comprobar esto último, se utilizaron los datos de espaciamiento de dos líneas de muestreo combinadas (Tz-L1d y Tz-L2d) que incluyen a fracturas F1 y F2. La Figura 10 muestra la formación de dos patrones distintivos en los gráficos de suma de correlación normalizada (SCN). Por un lado, se observa la formación de una pendiente relacionada con una distribución fractal de los grupos de fracturas (Fracturas F1). El otro patrón se asocia con la formación de una plataforma (y cuenca) sugiriendo que los grupos de fracturas están asociados a sitios de deformación localizada (Marrett et al., 2018). Dicha localización

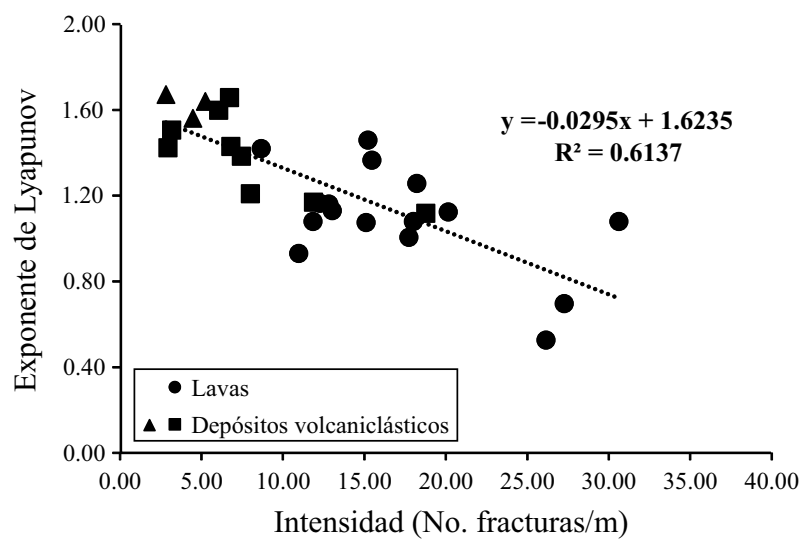

Figura 9. Relación entre el exponente de Lyapunov (grado de agrupamiento) y la intensidad de fracturas para los distintos tipos de roca que constituyen la sucesión del Mioceno. Es importante notar que las lavas (andesita o dacita) tienen una mayor intensidad con un menor grado de agrupamiento, mientras que los depósitos volcaniclásticos tienen un comportamiento contrario. 


\section{Líneas de muestreo con fracturas F1 y F2}

a)

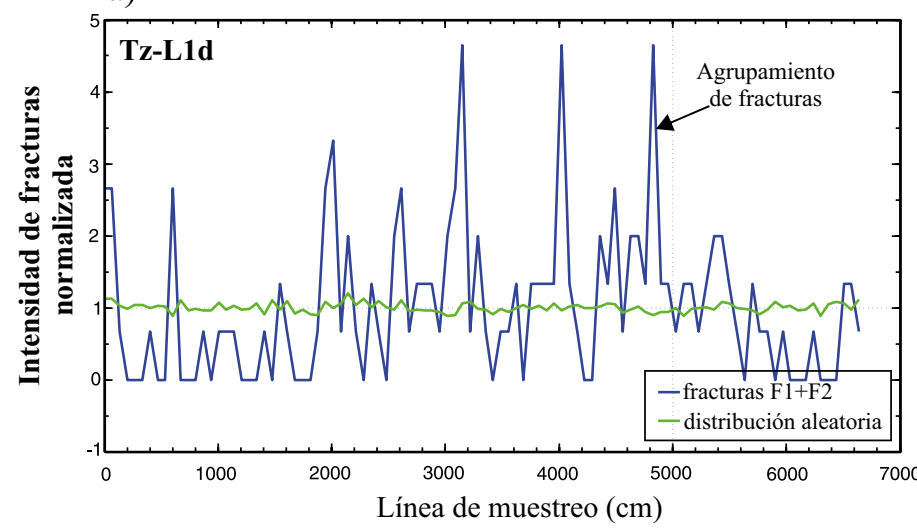

c)

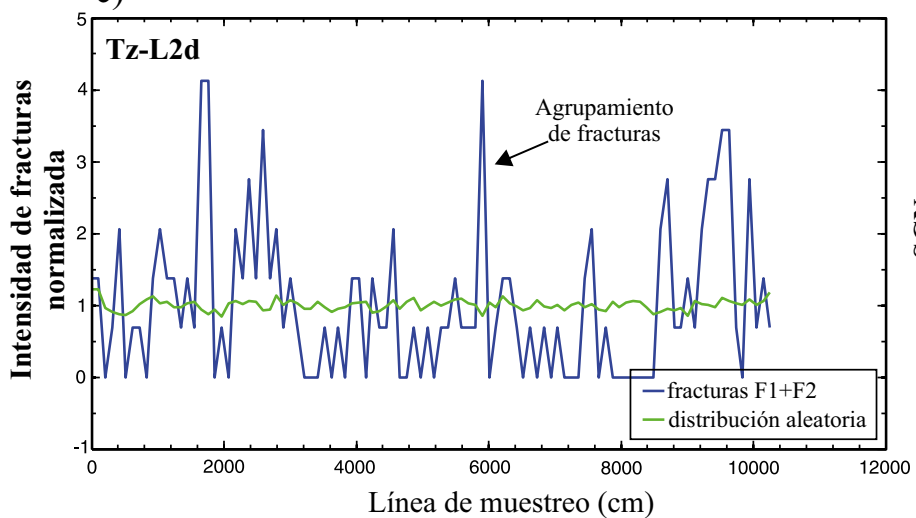

b)

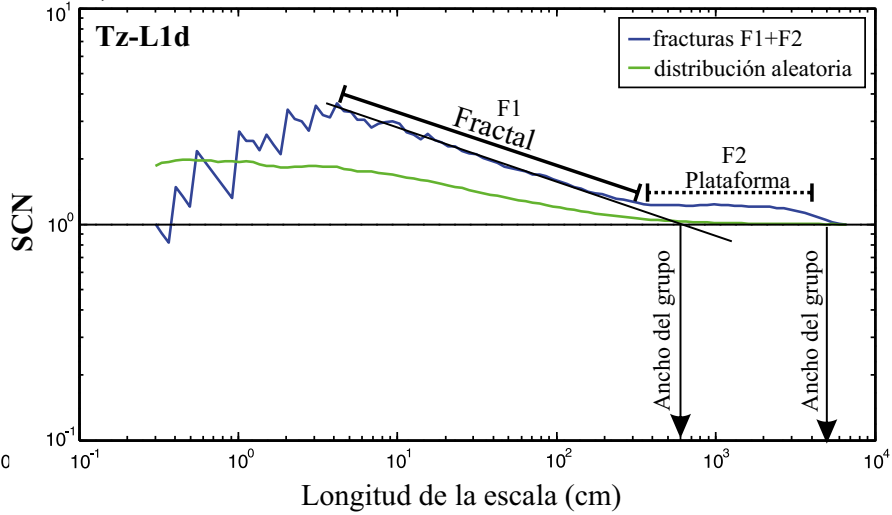

d)

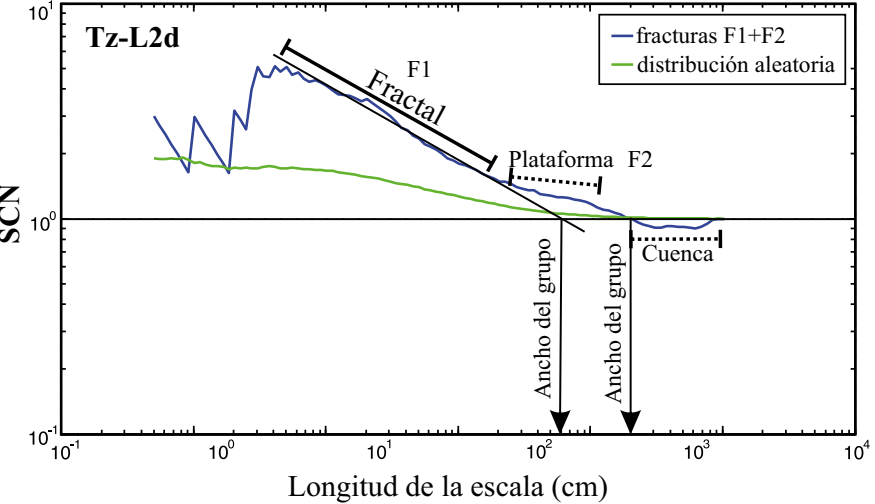

Figura 10. Gráficos de intensidad de fracturas normalizado (a, c) y suma de correlación normalizada (b, d) para 2 líneas de muestreo que incluyen a las fracturas F1 y F2. En las curvas de suma de correlación normalizada es posible apreciar la formación de dos patrones. En la longitud de escala menor es posible apreciar una distribución fractal de los grupos de fracturas asociada con las fracturas F1; mientras que el patrón de plataforma y/o cuenca en escalas mayores se relaciona con la presencia de grupos de fracturas de la familia F2.

puede estar relacionada con una herencia estructural o reactivación ligada a una anisotropía mecánica en rocas subyacentes (foliación, fracturas, fallas, pliegues) (Figura 11). La separación en los patrones observados en la Figura 11, se asocia con la inclusión de datos de espaciamiento de fracturas que incluyen a dos familias (F1 y F2), con dos comportamientos diferentes relacionados con el origen de cada familia de fracturas. En la zona de la cuenca de México, se tienen registrados por lo menos tres eventos de deformación que generan estructuras con orientaciones paralelas a las fracturas F2 (Figura 11). El evento más antiguo es un evento de extensión durante el Jurásico Tardío-Cretácico Temprano asociado con fallas NNW-SSE (Martini y Ortega-Gutiérrez, 2018). El segundo evento corresponde a un acortamiento cortical que desarrolló pliegues, cabalgaduras y foliación. Estas estructuras pertenecen al Orógeno Mexicano (Cretácico Tardío-Paleógeno), el cual tiene un rumbo preferencial NNW-SSE en el centro de México (Fitz-Díaz et al., 2018). Finalmente un tercer evento, se asocia con actividad de fallas laterales con cinemática derecha durante el Eoceno-Oligoceno (Alaniz-Álvarez et al., 2002). Todos estos eventos tectónicos generaron una anisotropía mecánica en rocas pre-miocénicas que influye en la localización, distribución y arreglo de las fracturas F2.

Las familias de fracturas que afectan a rocas del Mioceno en la cuenca de México están asociadas a tres eventos de deformación. Las fracturas F1 se generaron en un evento que involucra fallas de desplazamiento lateral derecho NE-SW activas en el Mioceno (Vásquez-Serrano et al., 2018b). Esta familia se caracteriza por formar grupos de fracturas que tienen una distribución fractal relacionada con la formación de una recta en el gráfico log-log de la longitud de la escala contra la suma de correlación normalizada (Figuras 9 y11). El segundo evento (Mioceno) se asocia con fallas normales que tienen un rumbo NNW-SSE, las cuales formaron fracturas (F2) cuyo arreglo se relaciona con herencia estructural o reactivación de estructuras previas (Figura 11). Esto es soportado con los gráficos de suma de correlación normalizada y con la orientación que tienen las fracturas F2. Finalmente, un evento de extensión N-S genera fallas normales que son activas actualmente y que produce una serie de fracturas (F3) que tienen un arreglo agrupado aparentemente con una distribución aleatoria en, por lo menos, dos órdenes de magnitud de escala (Figuras 10 y 11).

Con base en lo anterior, es posible sugerir que algunos parámetros fractales, en especial la suma de correlación normalizada, pueden ayudar a separar distintos tipos de familias de fracturas (sumado a su orientación) tomando en cuenta su arreglo. Adicionalmente, con la distribución espacial de los grupos de fracturas es posible conocer su relación con heterogeneidades del basamento y probablemente establecer su origen ligado con la neo formación y reactivación de fallas corticales. Para este último aspecto, será importante en el futuro hacer más estudios, como el que se presenta en este trabajo, en rocas del Mioceno dentro de la Faja Volcánica Transmexicana para encontrar las características (parámetros fractales) comunes que comparten las distintas familias fracturas, con el fin de establecer con precisión la relación entre el arreglo de las fracturas con su origen tectónico. 


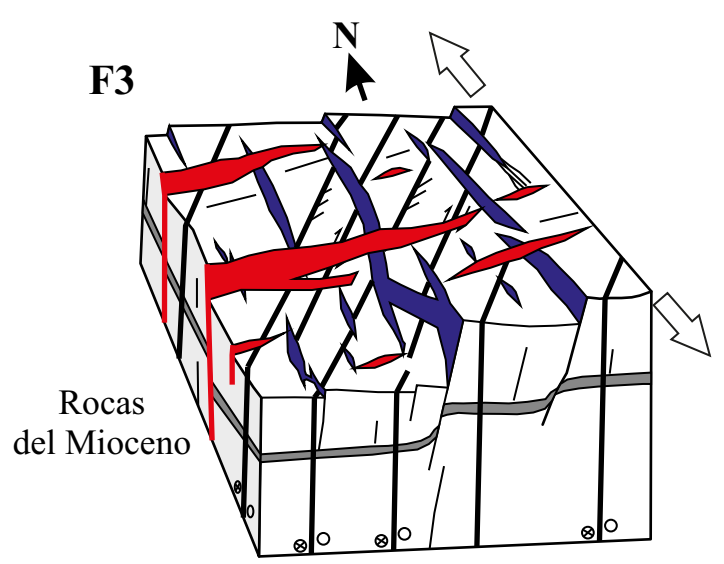

\section{Arreglo de fracturas}
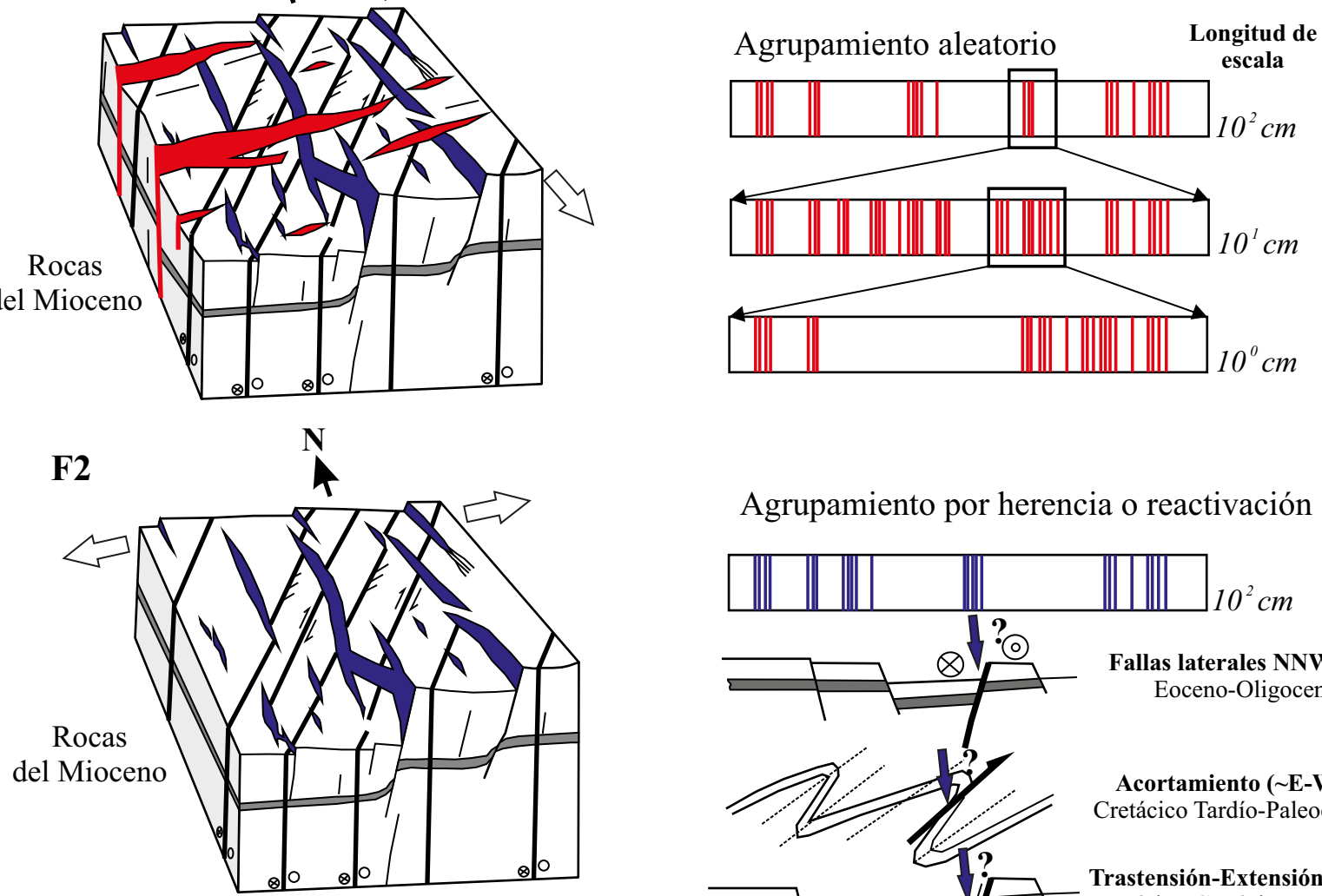

Agrupamiento por herencia o reactivación
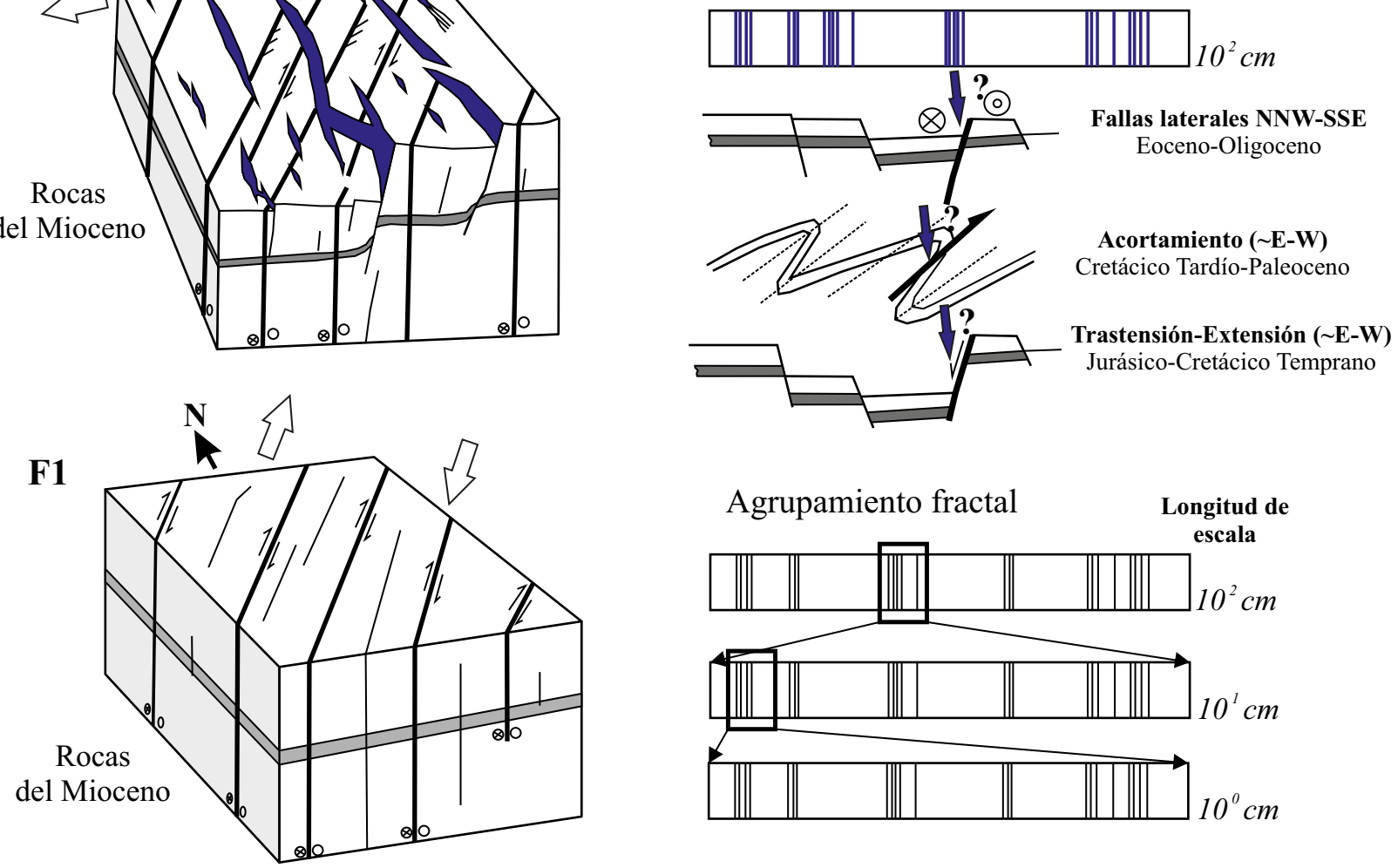

Figura 11. Modelos esquemáticos de la formación y arreglo de fracturas en rocas del Mioceno dentro de la cuenca de México. Las fracturas F1 se relacionan con la actividad de fallas laterales derechas con rumbo NE-SW que forman grupos de fracturas con una distribución fractal. Por otro lado, las fracturas F2 están asociadas con fallas normales (NNW-SSE) y forman grupos que se relacionan con anisotropías de rocas pre-miocénicas mediante una herencia estructural y/o reactivación. Finalmente, las fracturas F3 de generan a partir de fallas normales que tienen una orientación $\sim \mathrm{E}-\mathrm{W}$, formando grupos que tienen una distribución aleatoria.

\section{CONCLUSIONES}

Las fracturas que afectan a rocas del Mioceno, y que afloran dentro y en los alrededores de la cuenca de México, se pueden dividir en tres familias (F1, F2 y F3) con base en su orientación y en los eventos de deformación quebradiza reportados para el centro de México. Las fracturas F1 son las más antiguas y se asocian con fallas laterales (NESW), mientras que las fracturas F2 y F3 son las más jóvenes y están relacionadas con fallas normales NNW-SSE y E-W, respectivamente. Todas las fracturas tienden a formar grupos, donde el grado de agru- pamiento depende, en gran medida, del tipo de roca que afectan. Las lavas (andesitas y dacitas) tienen fracturas con menos grado de agrupamiento en comparación con los depósitos volcaniclásticos. Estos últimos están afectados por fracturas que forman grupos mejor definidos. Cada familia de fracturas tiene una distribución espacial particular en sus grupos de fracturas, para las fracturas F1 los grupos tienen un comportamiento fractal, mientras que los grupos de la familia F2 están asociados a una fábrica estructural en rocas pre-miocénicas. Finalmente las fracturas $\mathrm{F} 3$ tienen un arreglo agrupado que se relaciona con una distribución aleatoria. 


\section{AGRADECIMIENTOS.}

Agradecemos a Diana Marlene Flores, Miguel Ángel Meza Flores y Jesús Morales Pérez Vargas por su apoyo en el trabajo de campo. Este trabajo fue financiado por el proyecto PAPIIT IN101620 (a cargo del Dr. José Luis Arce Saldaña). Agradecemos los valiosos comentarios y correcciones del Dr. Gustavo Tolson, Dr. Shunshan Xu y de un revisor anónimo. Así mismo agradecemos las correcciones al manuscrito del Editor Dr. Ángel Francisco Nieto Samaniego. Todos los comentarios, sugerencias y correcciones ayudaron en gran medida a mejorar sustancialmente el manuscrito.

\section{REFERENCIAS}

Agustín-Flores, J., Siebe, C., Guilbaud, M.N., 2011, Geology and geochemistry of Pelagatos, Cerro del Agua, and Dos Cerros monogenetic volcanoes in the Sierra Chichinautzin Volcanic Field, south of México City: Journal of Volcanology and Geothermal Research, 201, 143-162.

Alaniz-Álvarez, S.A., Nieto-Samaniego, A.F., 2005, El sistema de fallas TaxcoSan Miguel de Allende y la Faja Volcánica Transmexicana, dos fronteras tectónicas del centro de México activas durante el Cenozoico: Boletín de la Sociedad Geológica Mexicana, Volumen Conmemorativo del Centenario, Grandes Fronteras Tectónicas de México, LVII(1), 65-82.

Alaniz-Álvarez, S.A., Nieto-Samaniego, A.F., Ferrari, L., 1998, Effect of the strain rate in the distribution of monogenetic and polygenetic volcanism in the Transmexican Volcanic Belt: Geology, 26, 591-594.

Alaniz-Álvarez, S.A., Nieto-Samaniego, A.F., Reyes-Zaragoza, M.A., OrozcoEsquivel, M.T., Ojeda-García, A.C., Vasallo-Morales, L.F., 2001, Estratigrafía y deformación de la región San Miguel de Allende-Querétaro: Revista Mexicana de Ciencias Geológicas, 18(1), 129-148.

Alaniz-Álvarez, S.A., Nieto-Samaniego, A.F., Orozco-Esquivel, M.T., VasalloMorales, L.F., Xu, S.S., 2002, El Sistema de Fallas Taxco- San Miguel de Allende: Implicaciones en la deformación post-Eocénica del centro de México: Boletín de la Sociedad Geológica Mexicana, 55, 12-29.

Arce, J.L., Layer P.W., Macías J.L., Morales-Casique, E., García-Palomo, A., Jiménez-Domínguez, F.J., Benowitz, J., Vásquez-Serrano, A., 2019, Geology and stratigraphy of the Mexico basin (Mexico city), central Trans-Mexican volcanic Belt: Journal of Maps, 15(2), 320-332.

Arce, J.L., Layer, P., Martínez, I., Salinas, J.I., Macías-Romo, M.C., MoralesCacique, E., Benowitz, J., Escolero, O.A., Lenhardt, N., 2015, Geología y estratigrafía del pozo profundo San Lorenzo Tezonco y sus alrededores, sur de la Cuenca de México: Boletín de la Sociedad Geológica Mexicana, 67(2), 123-143.

Arce, J.L., Layer P.W., Morales-Casique, E., Benowitz, J.A., Rangel, E., Escolero, O.A., 2013, New constraints on the subsurface geology of the Mexico City Basin: The San Lorenzo Tezonco deep well, on the basis of ${ }^{40} \mathrm{Ar} /{ }^{39} \mathrm{Ar}$ geochronology and whole-rock chemistry: Journal of Volcanology and Geothermal Research, 266, 34-49.

Arce, J.L., Macías, R., García-Palomo, A., Capra, L., Macías, J.L., Layer, P., Rueda, H., 2008, Late Pleistocene flank collapse of Zempoala Volcano (Central Mexico) and the role of fault reactivation: Journal of Volcanology and Geothermal Research, 177, 944-958.

Avendaño-Villeda, D. A., Caballero, M., Ortega-Guerrero, B., Lozano-García, S., Brown, E., 2018, Condiciones ambientales a finales del Estadio Isotópico 6 (EI 6:> 130000 años) en el centro de México: caracterización de una sección de sedimentos laminados proveniente del Lago de Chalco: Revista Mexicana de Ciencias Geológicas, 35(2), 168-178.

Barton, C.C., LaPointe, P.R., 1995, Fractal Analysis of Scaling and Spatial Clustering of Fractures, en Barton, C., La Pointe, P.R. (eds.), Fractals in Earth Science: New York, Plenum Press, 141-178.

Bear, J., Tsang, C.F., De Marsily, G., 2012, Flow and Contaminant Transport in Fractured Rock: San Diego, CA, USA, Academic Press, 560 pp.

Bloomfield, K., 1975, A late Quaternary monogenetic volcano field in central Mexico: Geologische Rundschau, 64, 476-497.

Bonnet, E., Bour, O., Odling, N.E., Davy, P., Main, I., Cowie, P., Berkowitz, B., 2001, Scaling of fracture systems in geological media: Reviews of Geophysics, 29, 347-383, https://doi.org/10.1029/1999RG000074.
Brogi, A., 2011, Variation in fracture patterns in damage zones related to strike-slip faults interfering with pre-existing fractures in sandstone (Calcionearea, southern Tuscany, Italy): Journal of Structural Geology, 33, 644-661.

Brown, E.T., Werne, J.P., Lozano-García, M.S., Caballero-Miranda, M., Ortega-Guerrero, B., Cabral-Cano, E., Valero-Garcés, B.L., Schwalb, A., Arciniega-Ceballos, A., 2012, Scientific Drilling in the Basin of Mexico to EvaluateClimate History, Hydrological Resources, and Seismic and Volcanic Hazards: Scientific Drilling, 14, 72-75, doi:10.2204/iodp. sd.14.12.2012.

Cadoux, A., Missenard, Y., Martínez-Serrano, R., Guillou, H., 2011, Trenchward Plio-Quaternary volcanism migration in the Trans-Mexican Volcanic Belt: the case of the Sierra Nevada range: Geological Magazine, 148(3), 492-506 doi:10.1017/S0016756810000993.

Cladouhos T.T., Marrett, R., 1996, Are fault growth and linkage models consistent with power-law distributions of fault lengths?: Journal of Structural Geology, 18, 281-293.

Cox, D.R., Lewis, P.A.W., 1966, The statistical analysis of series of events: London, Methuen's Monographs on Applied Probability and Statistics, $285 \mathrm{pp}$.

Davis, G. H., Reynolds, S.J., 1996, Structural geology of rocks and regions: New Jersey, Jhon Wiley \& Sons Inc., 212 pp.

Davy, P., Sornette, A., Sornette, D., 1990, Some consequences of a proposed fractal nature of continental faulting: Nature, 348, 56-58.

de Cserna, Z., de la Fuente-Duch, M., Palacios-Nieto, M., Triay, L., MitreSalazar, L.M., Mota-Palomino, R., 1988, Estructura geológica, gravimetría, sismicidad y relaciones neotectónicas regionales de la Cuenca de México: México, D.F., Universidad Nacional Autónoma de México, Instituto de Geología, Boletín 104, 71 pp.

Delgado-Granados, H., Nieto-Obregón, J., Silva-Romo, G., Mendoza- Rosales, C., Arellano-Gil, J., Lermo-Samaniego, J.F., Rodríguez- González., M., 1995, La Pera detachment fault system: active faulting south of Mexico City: geological evidence (II), en Reunión Anual de la Unión Geofísica Mexicana: Puerto Vallarta, Jalisco, GEOS, Resumen, 15(2), 64.

Ferrari, L., Orozco-Esquivel, T., Manea, V., Manea, M., 2011, The dynamic history of the Trans-Mexican Volcanic Belt and the Mexico subduction zone: Tectonophysics, 522-523, 122-149.

Fitz-Díaz, E., Tolson, G., Hudleston, P., Bolaños-Rodríguez, D., Ortega-Flores, B., Vásquez-Serrano, A., 2012, The role of folding in the development of the Mexican fold-and-thrust belt: Geosphere, 8(4), 931-949.

Fitz-Díaz, E., Lawton, T. F., Juárez-Arriaga, E., Chávez-Cabello, G., 2018, The Cretaceous-Paleogene Mexican orogen: Structure, basin development, magmatism and tectonics: Earth-Science Reviews, 183, 56-84.

Fossen, H., 2010, Structural geology, First Edition: New York, Cambridge University Press, $481 \mathrm{pp}$.

Fries, C., 1956, Bosquejo Geológico de la región entre México, D.F. y Taxco Guerrero, en $20^{\circ}$ Congreso Geológico Internacional: México, D.F., Libreto de las excursiones, Excursión A-9 y C-12, 11 pp.

Fries, C., 1960, Geología del Estado de Morelos y de partes adyacentes de México y Guerrero: Región central meridional de México: Universidad Nacional Autónoma de México, Boletín del Instituto de Geología, 60, 234 pp.

García-Palomo, A., Macías, J.L., Garduño, V.H., 2000, Miocene to Recent structural evolution of the Nevado de Toluca volcano region, Central Mexico: Tectonophysics, 318, 281-302.

García-Palomo, A., Carlos-Valerio, V., López-Miguel, C., Galván-García, A., Concha-Dimas A., 2006, Landslide inventory map of Guadalupe Range, north of the Mexico Basin: Boletín de la Sociedad Geológica Mexicana, Número especial de Geología Urbana, LVIII(2), 195-204.

García-Palomo, A., Zamorano, J.J., López-Miguel, C., Galván-García, A., CarlosValerio, V., Ortega, R., Macías, J.L., 2008, El arreglo morfoestructural de la Sierra de Las Cruces, México central: Revista Mexicana de Ciencias Geológicas, 25(1), 158-178.

Gillespie, P.A., Howard, C.B., Wash J.J., Watterson J., 1993, Measurement and characterization of spatial distributions of fractures: Tectonophisics, 226. 113-141.

González-Torres E. A., Morán-Zenteno D. J., Mori, L., Martiny B. M., 2015, Revisión de los últimos eventos magmáticos del Cenozoico del sector norte-central de la Sierra Madre del Sur y su posible conexión con el subsuelo profundo de la Cuenca de México: Boletín de la Sociedad 
Geológica Mexicana, 67, 285-297.

Guilbaud, M.-N., Siebe, C., Agustín-Flores, J., 2009, Eruptive style of the young high-Mg basaltic-andesite Pelagatos scoria cone southeast of México City: Bulletin of Volcanology, 71, 859-880.

Holder, J., Olson, J. E., Philip, Z., 2001, Experimental determination of subcritical crack growth parameters in sedimentary rock: Geophysical Research Letters, 28, 599-602.

Hooker, J.N., Laubach, S.E., Marrett, R., 2013, Fracture-aperture sized frequency, spatial distribution, and growth processes in strata-bounded and nonstratabounded fractures, Cambrian Meson Group, NW Argentina: Journal of Structural Geology, 54, 54-71.

Hooker, J.N., Laubach, S.E., Marrett, R., 2018, Microfracture spacing distributions and the evolution of fracture patterns in sandstones: Journal of Structural Geology, 108, 66-79.

La Pointe, P.R., Hudson, J.A., 1985, Characterization and interpretation of rock mass joint patterns: Geological Society of America, 199, https://doi. org/10.1130/SPE199-p1.

Laubach, S.E., Lamarche, J., Gauthier, B.D.M., Dunne, W.M., Sanderson, D.J., 2018, Spatial arrangement of faults and opening-mode fractures: Journal of Structural Geology, 108, 2-15.

Lenhardt, N., Böhnel, H., Wemmer, K., Torres-Alvarado, I. S., Hornung, J., Hinderer, M., 2010, Petrology, magnetostratigraphy and geochronology of the Miocene volcaniclastic Tepoztlán Formation: implications for the initiation of the Transmexican Volcanic Belt (Central Mexico): Bulletin of Volcanology, 72, 817-832.

Lermo, J.F., Santoyo, M.A., Jaimes, M.A., Antayhua, Y., Chavacán, M., 2016, Local Earthquakes of México Basin in Mexico City: k, Q, Source Spectra, and Stress Drop: Bulletin of the Seismological Society of America, 106(4), 1423-1437.

Lezama-Campos, J.L., Morales-Casique, E., Castrejón-Pineda, R., Arce, J.L., Escolero, O.A., 2016, Interpretación del registro geofísico del pozo profundo San Lorenzo Tezonco y su correlación litológica en la cuenca de México: Revista Mexicana de Ciencias Geológicas, 33(2), 198-208.

Lozano-Barraza, L., 1968, Geología de la Sierra de Guadalupe: México, D.F., Instituto Politécnico Nacional, tesis de licenciatura, 39 pp.

Lozano-García, M.S., Ortega-Guerrero, B., 1998, Late Quaternary environmental changes of the central part of the Basin of Mexico; correlation between Texcoco and Chalco basins: Review of Palaeobotany and Palynology, 99, 77-93.

Lozano-García, M.S., Ortega-Guerrero, B., Caballero-Miranda, M., UrrutiaFucugauchi, J., 1993, Late Pleistocene and Holocene paleoenvironments of Chalco Lake, central Mexico: Quaternary Research, 40, 332-342.

Lozano-García, S., Brown, E.T., Ortega-Guerrero, B., Caballero, M., Werne, J., Fawcett, P.J., Schwalb, A., Valero-Garcés, B., Schnurrenberger, D., O'Grady, R., Stockhecke, M., Steinman, B., Cabral-Cano, E., Caballero C., Sosa-Nájera, S., Soler, A.M., Pérez, L., Noren, A., Myrbo, A., Bücker, M., Wattrus, N., Arciniega, A., Wonik, T., Watt, S., Kumar, D., Acosta, C., Martínez, I., Cosío, R., Ferland, T., Vergara-Huerta, F., 2017, Perforación profunda en el lago de Chalco: reporte técnico: Boletín de la Sociedad Geológica Mexicana, 69(2), 299-311.

Macías, J.L., Arce, J.L., García-Tenorio, F., Layer, P.W., Rueda, H., Reyes-Agustín, G., López-Pizaña, F., Avellán, D., 2012, Geology and geochronology of Tlaloc, Telapón, Iztaccíhuatl, and Popocatépetl volcanoes, Sierra Nevada, central Mexico, en Aranda-Gómez, J.J., Tolson, G., Molina-Garza, R.S. (eds.), The Southern Cordillera and Beyond: Geological Society of America Field Guide, 25, 163-193.

Mandelbrot, B.B., 1967, How long is the coast of Britain? Statistical self-similarity and fractional dimension: Science, 156, 636-638.

Manea, V.C., Manea, M., Ferrari, L., 2013, A geodynamical perspective on the subduction of Cocos and Rivera plates beneath Mexico and Central America: Tectonophysics, 609, 56-81.

Márquez, A., Oyarzu, R., Doblas, M., Verma, S.P., 1999, Alkaline (oceanic island basalt type) and calc-alkaline volcanism in the Mexican Volcanic Belt: A case for plume-related magmatism and propagating rifting at an active margin?: Geology, 27, 51-54.

Marrett, R., Ortega, O.J., Kelsey, C.M., 1999, Extent of power-law scaling for natural fractures in rock: Geology, 27, 799-802.

Marrett, R., Gale, J.F., Gómez, L.A., Laubach, S.E., 2018, Correlation analysis of fracture arrangement in space: Journal of Structural Geology, 108, 16-33.
Marshak, S., Mitra, G., 1988, Basic Methods of Structural Geology: New Jersey, Pretice-Hall. Inc., 446 pp.

Martínez-Abarca, R., Lozano-García, S., Ortega-Guerrero, B., CaballeroMiranda, M., 2019, Incendios y actividad volcánica: historia de fuego en la cuenca de México en el Pleistoceno tardío con base en registros de material carbonizado en el lago de Chalco: Revista Mexicana de Ciencias Geológicas, 36(2), 259-269.

Martini, M., Ortega-Gutiérrez, F., 2018, Tectono-stratigraphic evolution of eastern Mexico during the break-up of Pangea: A review: Earth-Science Reviews, 183, 38-55.

Mooser, F., 1963, Historia tectónica de la Cuenca de México: Boletín de la Asociación Mexicana de Geólogos Petroleros, 15, 239-245.

Mooser, F., 1975, Historia geológica de la Cuenca de México, en Memoria de las obras de drenaje profundo del Distrito Federal: México, D.F., Departamento del Distrito Federal, 38 pp.

Morales-Casique, E., Escolero, O.A., Arce, J.L. 2014, Resultados del pozo San Lorenzo Tezonco y sus implicaciones en el entendimiento de la hidrogeología regional de la cuenca de México: Revista Mexicana de Ciencias Geológicas, 31(1), 64-75.

Morales-Casique, E., Arce-Saldaña J.L., Escolero-Fuentes O., Lezama-Campos J.L., 2016, Análisis de la estratigrafía y las características hidrogeológicas de los estratos profundos que conforman el subsuelo de la Cuenca de México a partir de la perforación de dos pozos profundos, uno a $2000 \mathrm{~m}$ y otro a 1570 m denominados Agrícola Oriental 2B y 2C respectivamente: México, D.F., México, Reporte técnico para el Sistema de Aguas de la Ciudad de México, Gobierno de la Ciudad de México, 178 pp.

Morales-Casique, E., Arce-Saldaña J.L., Escolero O.A., Lezama-Campos, J.L., 2017, Analizar la estratigrafía y las características hidrogeológicas de los estratos profundos que conforman el subsuelo de la Cuenca de México a partir de la perforación a $2000 \mathrm{~m}$ del pozo profundo denominado "Santa Catarina 3": Ciudad de México, México, Reporte técnico para el Sistema de Aguas de la Ciudad de México, Gobierno de la Ciudad de México, 113 pp.

Nieto-Samaniego, A.F., Alaniz-Álvarez, S., Tolson, G., Oleschko, K., Korvin. G., Xu, S.S., Pérez- Venzor, J.A., 2005, Spatial Distribution, Scaling and Self-similar Behavior of Fracture Arrays in the Los Planes Fault, Baja California Sur, México: Pure Applied Geophysics, 162(2005), 805-826.

Nieto-Samaniego, A.F., Alaniz-Álvarez, S., Tolson, G., Xu, S., Pérez-Venzor, A., 2003, Estimación de densidades, distribuciones de longitud y longitud total de fracturas: un caso de estudio en la Falla de Los Planes, La Paz, B.C.S.: Boletín de la Sociedad Geológica Mexicana, LVI(1), 1-9.

Norini, G., Groppelli, G., Lagmay, A.M.F., Capra, L., 2006, Recent left oblique slip faulting in the central eastern Trans-Mexican Volcanic Belt: Seismic hazard and geodynamic implications: Tectonics, 25(4), TC4012, 1-21, doi: $10.1029 / 2005$ TC001877.

Odling, N. E., Gillespie, P. A., Bourgine, B., Castaing, C., Chils, J. P., Christensen, N. P., Fillion, E., Genter, A., Olsen, C., Thrane, L., Trice. R., Aarseth, E., Walsh. J. J., Watterson, J., 1999, Variations in fracture system geometry and their implications for fluid flow in fractured hydrocarbon reservoirs: Petroleum Geoscience, 5, 373-384.

Olson, J.E., 1993, Joint pattern devenlopment: effects of subcritical crack growth an mechanical crack interaction: Journal of Geophisical Research, 98, 12251-12265.

Olson, J.E., 2003, Sublinear scaling of fracture aperture versus length: An exception or the rule?: Journal of Geophysical Researh, Solid Earth, 108(B9), 2413, doi:10.1029/2001JB000419.

Olson, J., Pollard, D.D., 1989, Inferring paleostresses from natural fracture patterns: A new method: Geology, 17(4), 345-348.

Osete, M.L., Ruíz-Martínez, V.C., Caballero, M.C., Galindo, C., UrrutiaFucugauchi, J., Tarling, H.D., 2000, Southward migration of continental volcanic activity in the Sierra de Las Cruces, Mexico: Paleomagnetic and radiometric evidence: Tectonophysics, 318, 201-215.

Pardo, M., Suárez, G., 1995, Shape of the subducted Rivera and Cocos plates in southern Mexico: Seismic and tectonic implications: Journal of Geophysical Research, 100(87), 12357-12373.

Pérez-Cruz, G.A., 1988, Estudio sismológico de reflexión del subsuelo de la ciudad de México: México, D.F., Universidad Nacional Autónoma de México, tesis de maestría, 83 pp.

Ramsay, J.G., 1967, Folding and Fracturing of Rocks: New York, McGraw-Hill, 568 pp. 
Ramsay, J.G., Huber, M.I., 1987, The Techniques of Modern Structural Geology, Vol. 2. Folds and Fractures: London, Academic Press, 391 pp.

Ramsey, J.M., Chester, F.M., 2004, Hybrid fracture and the transition from extension fracture to shear fracture: Nature, 428(6978), 63-66.

Riley, P., Tikoff, B., Murray, A.B., 2011, Quantification of fracture networks in non-layered, massive rock using synthetic and natural data sets: Tectonophysics, 505, 44-56.

Rosenstein, M.T., Collins, J.J., De Luca, C.J., 1993, A practical method for calculating largest Lyapunov exponents from small data sets: Physica, D, Nonlinear Phenomena, 65(1-2), 117-134, https://doi. org/10.1016/0167-2789(93)90009-P.

Samsu, A., Cruden, A.R., Micklethwaite, S., Grose, L., Vollgger, S.A., 2020, Scale matters: The influence of structural inheritance on fracture patterns: Journal of Structural Geology, 130, 103896.

Siebe, C., 2000, Age and archaelogical implications of Xitle volcano, southwestern Basin of Mexico City: Journal of Volcanology and Geothermal Research, 104, 45-64.

Siebe, C., Arana-Salinas, L., Abrams, M., 2005, Geology and radiocarbon ages of Tláloc, Tlacotenco, Cuauhtzin, Hijo del Cuauhtzin, Teuhtli, and Ocusacayo monogenetic volcanoes in the central part of the Sierra Chichinautzin, México: Journal of Volcanology and Geothermal Research, 141, 225-243.

Sunye-Puchol, I., Lacan, P., Ontuño, M., Villamor, P., Audin, L., Zúñiga, F.R., Langridge, R.M., Aguirre-Díaz G. de J., Lawton, T.F., 2015, La falla San Mateo: nuevas evidencias paleosismológicas de fallamiento activo en el graben de Acambay, México: Revista Mexicana de Ciencias Geológicas, 32(3), 361-376.

Suter, M., López-Martínez, M., Quintero-Legorreta, O., Carrillo-Martínez, M., 2001, Quaternary intra-arc extension in the central Trans-Mexican volcanic belt: Geological Society of America Bulletin, 113(6), 693-703.

Vásquez-Serrano A., 2013, Análisis fractal de fracturas geológicas en un análogo expuesto de yacimientos carbonatados naturalmente fracturados y su aplicación en modelos de flujo de fluidos: México. D.F., México, Universidad Nacional Autónoma de México, tesis de maestría. 153 pp.
Vásquez-Serrano, A, Tolson, G., Fitz-Diaz, E., Chávez-Cabello, G., 2018a, Influence of pre-tectonic carbonate facies architecture on deformation patterns of syntectonic turbidites, an example from the central Mexican fold-thrust belt: Journal of Structural Geology, 109, 127-139.

Vásquez-Serrano, A., Arce, J. J., Arroyo, S., Morales, E., Camacho, R., 2018b Distribución y arreglo de fracturas en el centro de la cuenca de México, en Reunión Anual Unión Geofísica Mexicana: Puerto Vallarta Jalisco, Unión Geofísica Mexicana, GEOS 38(1), Resumen.

Vásquez-Serrano, A., Camacho-Rangel, R., Arce-Saldaña, J. L., Morales-Casique, E., 2019, Análisis de fracturas geológicas en el pozo Agrícola Oriental 2C, Ciudad de México y su relación con fallas mayores: Revista Mexicana de Ciencias Geológicas, 36(1), 38-53.

Wallace, P., Carmichael, I.S.E., 1999, Quaternary volcanism near the Valley of Mexico: implications for subduction zone magmatism and the effects of crustal thickness variations on primitive magma compositions: Contributions to Mineralogy and Petrology, 135, 291-314.

Wang, Q., Laubach, S. E., Gale, J. F. W., Ramos, M. J., 2019, Quantified fracture (joint) clustering in Archean basement, Wyoming: application of the normalized correlation count method: Petroleum Geoscience, 25(4), 415-428.

Manuscrito recibido: junio 10, 2020

Manuscrito corregido recibido: octubre 5, 2020

Manuscrito recibido: octubre 6, 2020 